INSTITUT NATIONAL DE LA STATISTIQUE ET DES ETUDES ECONOMIQUES

Série des Documents de Travail du CREST

(Centre de Recherche en Economie et Statistique)

\author{
$n^{\circ}$ 2006-04 \\ Modélisation dynamique de la \\ participation au marché du \\ travail des femmes en couple \\ C. EDON \\ T. KAMIONKA 2
}

Les documents de travail ne reflètent pas la position de l'INSEE et n'engagent que leurs auteurs.

Working papers do not reflect the position of INSEE but only the views of the authors.

\footnotetext{
${ }^{1}$ ERMES (CNRS), Université Panthéon-Assas (Paris 2); 12 Place du Panthéon, 75230 Paris Cédex 050 (cedon@u-paris2.fr)

${ }^{2}$ CNRS et CREST, Timbre J390, 15 Boulevard Gabriel Péri, 92245 Malakoff Cédex. (kamionka@ensae.fr) Les auteurs ont apprécié les suggestions faites par Olivier Bargain, Georges Bresson, Thibault Brodaty, Bernard Fortin, Joseph Lafranchi et Dominique Meurs, ainsi que les commentaires des participants des 22èmes Journées de Microéconomie Appliquée et du Congrès de l’AFSE (2005).
} 


\title{
Modélisation dynamique de la participation au marché du travail des femmes en couple
}

\author{
Cyriaque Edon, $\quad$ Thierry Kamionka ${ }^{\dagger}$
}

Janvier 2006

* Ermes (CNRS), Université Panthéon-Assas (Paris 2), 12 Place du Panthéon 75230 Paris Cédex 050 (cedon@u-paris2.fr).

${ }^{\dagger}$ CNRS et CREST. Timbre J390. 15, Boulevard Gabriel Péri. 92245 Malakoff Cedex. (kamionka@ensae.fr).

Les auteurs ont apprécié les suggestions faites par Olivier Bargain, Georges Bresson, Thibault Brodaty, Bernard Fortin, Joseph Lafranchi et Dominique Meurs, ainsi que les commentaires des participants des 22èmes Journées de Microéconomie Appliquée et du congrès de l'AFSE (2005). 


\title{
Résumé
}

Nous considérons une modélisation dynamique de la décision de participation au marché français du travail des femmes en couple pour la période allant de 1994 à 2001. Nous estimons, sur les données du volet français du panel Européen, par la méthode du maximum de vraisemblance simulée, un modèle probit dynamique à effet aléatoire sur une base mensuelle et annuelle en tenant compte du problème des conditions initiales. La décision de participation des femmes en couple dépend des caractéristiques individuelles et est caractérisée par une dépendance importante de l'état. Les composantes d'hétérogénéité non observables jouent un rôle central dans la dynamique de la participation. Ces composantes non observables reflètent des différences au niveau des préférences individuelles concernant l'arbitrage consommation-loisir. Par ailleurs, nous trouvons que les termes d'erreur du modèle sont corrélés négativement. Nous montrons l'importance de la composante du revenu permanent dans la décision de participation.

Mots Clés : Participation, hétérogénéité, estimation par simulation, données de panel.

Classification JEL : J21, J22, C33, C35.

\begin{abstract}
We consider a dynamic model for the participation decision to the labour market of the women who live in couple. We estimate a dynamic probit model with random effects using the french part of the Europanel, for the period going from 1994 to 2001 and using a simulated maximum likehood estimator. These estimates are made on a monthly and an annual basis taking into account the initial conditions problem. The decision of participation of the women who live in couple depends on the individual characteristics and is characterized by a significant state dependence. The unobserved components of heterogeneity play a central role in the dynamics of the participation to the labour market. This unobserved heterogeneity reflect differences in the preferences relative to the arbitrage between consumption and leisure. We find that the error terms of the model are negatively correlated. Moreover, we show the importance of the permanent income component in the participation decision.
\end{abstract}

Keywords : Participation, heterogeneity, simulated maximum likelihood estimator, panel data. 


\section{Introduction}

Dans cet article, nous étudions la dynamique de l'offre de travail des femmes vivant en couple. Il existe deux composantes de cette offre : la décision de participation et la durée de travail offerte. Plusieurs études de l'offre de travail ont montré que la première composante est la plus importante, dans la mesure où la durée de travail souhaitée est souvent contrainte par les entreprises. Par ailleurs, des auteurs ont souligné la difficulté de modéliser ces deux composantes lorsqu'il existe des coûts fixes associé à l'offre de travail (cf. Heckman (1993), Hyslop (1999)). Aussi, l'analyse de l'offre de travail des femmes doit-elle dégager en premier lieu les déterminants de leur participation au marché du travail.

Comme la décision de participation connaît une plus grande variabilité à la fois à travers les individus et au cours du cycle de vie chez les femmes en couple, nous avons choisi de restreindre notre étude aux seules femmes dans cette situation. En cela nous rejoignons les travaux pionniers sur l'offre de travail des femmes réalisés au cours des années soixante dix (cf. Heckman et Willis (1977, 1979), Mincer et Ofek (1979)).

Peu d'études dynamiques de la participation des femmes au marché du travail ont été faites sur des données de panel françaises. La raison réside essentiellement dans la difficulté de trouver des données adaptées à ce type d'étude. Les premières analyses ont été réalisées à partir des fichiers longitudinaux des enquêtes Emploi de l'Insee qui permettent d'observer la participation à trois dates successives séparées par un intervalle d'un an. Ainsi, Lollivier (1995) étudie le comportement d'activité des femmes en tenant compte de la présence d'une composante d'hétérogénéité. Lollivier (2001) a réalisé, dans un cadre statique, une étude de la participation des femmes à partir des quatre premières vagues de la partie française du panel européen. Il montre que ne pas tenir compte de l'hétérogénéité des individus conduit à surestimer l'effet de l'âge sur la décision de participation au marché du travail. Ce panel permet de suivre les individus dans le temps sur une période de temps relativement longue et de disposer d'un ensemble de caractéristiques individuelles, comme les naissances d'enfants ou les revenus des autres membres de la famille, particulièrement pertinentes pour l'analyse de la décision d'activité des femmes en couple.

Ces dernières années, les progrès réalisés sur le plan de la modélisation et des techniques d'estimation ont été tels qu'il est possible de considérer une modélisation dynamique de la décision de participation au marché du travail. Ainsi, on peut souligner, notamment, les apports réalisés par Geweke (1991), Hajivassiliou (1992) et Keane (1994) pour leur contribution à l'estimateur GHK et Heckman (1981) pour le traitement des conditions initiales. Ces avancées réalisées en économétrie permettent aujourd'hui, à partir d'un ensemble de données particulièrement riches, de considérer une prise en compte, dans le cadre d'une modélisation dynamique, de l'impact 
de l'hétérogénéité non observable sur la décision d'activité.

Le modèle que nous avons retenu pour décrire la participation des femmes est dynamique et permet d'obtenir une dépendance de l'état. En d'autres termes, la décision de participer au marché de travail dépend non seulement des caractéristiques individuelles - observées ou non - mais aussi des décisions de participation antérieures. Cette forme de dépendance peut résulter, en particulier, de coûts de recherche différents selon leur situation par rapport au marché du travail (cf. Eckstein et Wolpin (1990), Hyslop (1999)). Le modèle considéré dans cet article permet d'avoir une corrélation sérielle entre les termes de l'équation de participation permettant aux chocs transitoires sur l'offre de travail de se répercuter d'une période à l'autre. Notre modélisation comporte une prise en compte de l'hétérogénéité non observable sous la forme d'un effet individuel aléatoire. Cet effet individuel synthétise, par exemple, les différences non observables au niveau des préférences individuelles concernant l'arbitrage entre consommation et loisir. Parce que notre modèle incorpore de l'hétérogénéité non observable et que les individus sont observés pour la première fois après leur entrée dans la vie active, nous sommes amenés à modéliser les conditions initiales selon la méthode proposée par Heckman (1981). Notre modèle tient compte de la dépendance de l'état, de la dépendance sérielle et des conditions initiales. De plus, l'échantillon utilisé couvre une période de temps relativement longue (1994-2001).

Les résultats obtenus dans le cadre d'un modèle non dynamique - sans dépendance de l'état - sont biaisés. Comme le prévoit le modèle classique d'offre de travail, plus le niveau du diplôme est élevé, plus l'activité est importante. La présence d'un enfant va réduire l'activité et cela d'autant plus qu'il est jeune. La relation existant entre l'activité des femmes en couple et le revenu non salarial a un contenu économique moins intuitif. En effet, les femmes dont les revenus des conjoints sont relativement peu élevés ont une activité généralement moins importante que celle des femmes dont les revenus des conjoints appartiennent à la tranche de salaire immédiatement supérieure. Ce phénomène peut s'expliquer par l'existence d'une législation "fiscalo-sociale" qui n'incite pas les femmes à l'activité lorsque le conjoint a des revenus faibles. Pour les autres classes de revenu du conjoint on retrouve une relation entre revenu et activité telle qu'elle est prédite par la théorie classique de l'offre de travail et mise en évidence précédemment sur données américaines (cf. Hyslop 1999).

Nous faisons apparaître une dépendance sérielle significative entre les termes d'erreur du modèle même si elle est faible. Les résultats des estimations du modèle dynamique montrent que les conditions initiales sont particulièrement informatives de la distribution de l'hétérogénéité non observable.

Après la présentation du modèle (section 2), la troisième section décrit l'échantillon utilisé qui provient de la partie française de l'ECHP ("Europa- 
nel"). Les principaux résultats de l'étude sont présentés et discutés dans le cadre de la section 4 . Nous concluons dans la dernière section et évoquons quelques pistes de recherche.

\section{Spécification du modèle}

\subsection{Modélisation empirique}

Pour modéliser la participation au marché du travail des femmes, nous considérons la spécification dynamique en forme réduite suivante ${ }^{1}$ :

$$
y_{i t}=\mathbb{I}\left[x_{i t}^{\prime} \beta+\gamma y_{i t-1}+u_{i t}>0\right]
$$

où $y_{i t}$ vaut 1 si la personne participe au marché du travail et 0 dans le cas contraire $(t=1, \ldots, T$, et $i=1, \ldots, n)$. La première date de présence dans le Panel Européen est traitée spécifiquement. $x_{i t}$ est un vecteur de caractéristiques individuelles observables pouvant comprendre la situation matrimoniale, le revenu du conjoint, le niveau d'éducation, l'âge, les caractéristiques démographiques liées à la présence, éventuelle, d'enfants dans le ménage.

Nous considérerons ici que le seul revenu pris en compte dans la décision d'activité de la femme, en dehors du revenu salarial qu'elle obtient lorsqu'elle passe en emploi, est le revenu d'activité de son conjoint qui peut, selon le cas, comprendre les allocations chômage perçues par ce dernier. Ceci revient à ne pas prendre en compte les diverses prestations sociales qui dépendent du revenu total du ménage et du nombre d'enfants. Ce choix est fait pour éviter les problèmes d'endogénéité du revenu non salarial des femmes (comme par exemple l'Allocation Parentale d'Éducation introduite en 1994). Remarquons que ne pas prendre en compte les autres allocations familiales - celles attribuées sans conditions de ressources - dans la décision d'activité des femmes peut s'analyser comme si elles étaient incluses dans les composantes inobservées de la décision de participer ou non au marché du travail. Sachant qu'il n'y a pas eu de changement majeur sur la période considérée dans ces prestations, les résultats ne seraient pas sensiblement différents si l'on faisait apparaître cette composante dans le revenu du ménage.

$u_{i t}$ est la réalisation d'une variable aléatoire résumant, à la fois, l'ensemble des caractéristiques individuelles non observables constantes dans le temps et l'existence d'une composante résiduelle aléatoire variant à chaque date. $\beta$ est

\footnotetext{
${ }^{1}$ Cette spécification peut être obtenue à partir d'une modélisation structurelle de l'offre de travail (cf., par exemple, Hyslop 1999). Hyslop fait l'hypothèse que, pour trouver un emploi, l'individu doit nécessairement avoir une activité de recherche. Il suppose qu'il n'y a pas de recherche en emploi. Les individus sans emploi à la recherche d'un emploi font face à un coût recherche. La fonction d'utilité, supposée séparable dans le temps, dépend de la consommation du ménage et du nombre d'heures travaillées (constantes quelque soit l'emploi). Le choix d'activité du conjoint est supposé être exogène.
} 
un vecteur de paramètres qui regroupe les coefficients associés aux variables explicatives observables. $\gamma$ est un paramètre réel.

Le terme d'hétérogénéité de l'équation (1) a pour expression

$$
u_{i t}=\alpha_{i}+\epsilon_{i t},
$$

où $\alpha_{i}$ est une composante d'hétérogénéité individuelle non observable. Deux spécifications peuvent être retenue pour la distribution de $\alpha_{i}$. La première est telle que le terme $\alpha_{i}$ est indépendant de $x_{i t}$. Dans ce cas, on fait l'hypothèse que

$$
\alpha_{i}=\eta_{i},
$$

où les effets individuels $\eta_{i}, i=1, \ldots, n$, sont indépendants et identiquement distribués. La seconde spécification conduit à donner l'expression de $\alpha_{i}$ en fonction de caractéristiques individuelles et d'un terme d'erreur constant dans le temps. Il s'agit d'une spécification à effets aléatoires corrélés $(\mathrm{CRE})^{2}$ :

$$
\begin{gathered}
\alpha_{i}=\sum_{t=0}^{T}\left\{\xi_{1 t} \operatorname{Nbenf}[0-3]_{i t}+\xi_{2 t} \operatorname{Nbenf}[3-5]_{i t}+\xi_{3 t} \operatorname{Nbenf}[6-11]_{i t}\right. \\
\left.+\xi_{4 t} \operatorname{Nbenf}[12+]_{i t}\right\} \quad+\sum_{t=0}^{T-1} \xi_{5 t} y_{m i t}+\eta_{i}
\end{gathered}
$$

où $y_{\text {mit }}$ est l'écart à la moyenne du revenu salarial du conjoint de la femme à la date t. $N$ benf $[0-3]_{i t}, N$ benf $[3-5]_{i t}$, Nbenf $[6-11]_{i t}$ Nbenf $[12+]_{i t}$ sont des composantes de la fécondité de l'individu i qui désignent, respectivement, le nombre d'enfants âgés de moins de 3 ans, de 3 à 5 ans, de 6 à 11 ans et de plus de 12 ans à la date t. Les termes $\xi_{j t}(j=1, \ldots, 5)$, sont des paramètres à estimer.

Cette spécification permet de corréler la décision de participation avec les variables qui décrivent la fécondité. $\epsilon_{i t}$ est un terme aléatoire supposé être indépendant de $x_{i t}\left(\epsilon_{i, t}\right.$ est indépendant de $\epsilon_{i^{\prime}, t^{\prime}}$, si $\left.i \neq i^{\prime}\right)$. Comme Hyslop (1999), nous faisons l'hypothèse que la distribution de $\epsilon_{i t}$ a une structure autorégressive. Cette hypothèse va nous permettre d'évaluer l'effet d'un choc dans le temps sur les préférences individuelles qui va pouvoir se propager, en partie, d'une période à l'autre.

La distribution de l'effet individuel est telle que $\eta_{i}$ est supposé être indépendant des caractéristiques individuelles observables $x_{i t}$ et distribué selon une loi normale centrée de variance $\sigma_{\eta}^{2}$. On peut remarquer que la spécification usuelle à effets aléatoires indépendants (2) peut être considérée comme un cas particulier de celle à effets corrélés (3) dans le cas où $\xi_{j t}=0, \forall j, \forall t$. Aussi, cette dernière restriction pourra être testée.

Dans un tel modèle de panel dynamique à effets aléatoires comportant une censure à gauche, les conditions initiales doivent être modélisées avec

\footnotetext{
${ }^{2}$ Pour éviter les problèmes de colinéarité entre $y_{p}$ et le variables $y_{m t}$, le modèle est estimé en utilisant la restriction $\sum_{t=0}^{T-1} \xi_{5 t}=0$.
} 
attention. L'approche retenue est celle proposée par Heckman (1981) qui consiste à retenir pour les conditions initiales une spécification en forme réduite permettant de corréler le terme d'erreur de l'équation initiale avec les termes d'erreur ultérieurs.

Considérons la décision de participation à la date initiale

$$
y_{i 0}=\mathbb{I}\left[x_{i 0}^{\prime} \beta_{0}+u_{i 0}>0\right],
$$

où $x_{i 0}$ est le vecteur des caractéristiques individuelles initiales et $u_{i 0}$ est un terme d'erreur qui a la propriété de pouvoir être corrélé avec les termes $u_{i t}$, pour les dates $t=1, \ldots$, T. $\beta_{0}$ est le vecteur de paramètres associés aux variables explicatives qui figurent dans l'équation initiale.

En ce qui concerne le terme d'erreur de l'équation initiale dans le modèle, l'hypothèse suivante est faite

$$
u_{i 0} \sim \mathrm{N}\left(0, \sigma_{0}^{2}\right) .
$$

On adopte une structure autoregressive pour les termes d'erreur des équations ultérieures

$$
\epsilon_{i t}=\rho \epsilon_{i t-1}+\nu_{i t}
$$

où $\eta_{i} \Perp \nu_{i t}, t=1, \ldots, \mathrm{T}$, et

$$
\nu_{i t} \sim \mathrm{N}\left(0, \sigma_{\nu}^{2}\right) .
$$

D'autre part, comme cela a été proposé par Heckman (1981), les termes d'erreur initiaux sont tels que la corrélation entre $u_{i 0}$ et $u_{i t}$, pour $t=1, \ldots, \mathrm{T}$, est, a priori, non nulle.

\subsection{Identification}

La spécification considérée est celle d'un modèle probit dynamique à effet aléatoire. Un certain nombre d'hypothèses doivent être faites pour obtenir l'identification du modèle.

En effet, à chaque date, la variable endogène est dichotomique. Il en résulte que l'on est amené à supposer que

$$
\sigma_{\eta}^{2}+\sigma_{\epsilon}^{2}=1
$$

et

$$
\operatorname{var}\left(u_{i 0}\right)=\sigma_{0}^{2}=1 .
$$

On peut montrer que

$$
\operatorname{var}\left(\epsilon_{i t}\right)=\sigma_{\epsilon}^{2}=\frac{\sigma_{\nu}^{2}}{\left(1-\rho^{2}\right)} .
$$


Soit $\tilde{U}_{i}=\left(u_{i 1}, u_{i 2}, \cdots, u_{i T}\right)^{\prime}$, le vecteur des termes d'erreur des équations autres que celle relative à la date initiale. On obtient que

$$
\tilde{U}_{i} \sim \mathrm{N}\left(0, \Omega_{u}\right)
$$

où

$$
\Omega_{u}=\operatorname{var}\left(\tilde{U}_{i}\right)=\sigma_{\eta}^{2} \mathbb{1}_{T} \mathbb{1}_{T}^{\prime}+\frac{\sigma_{\nu}^{2}}{\left(1-\rho^{2}\right)}\left[\begin{array}{ccccc}
1 & \rho & \rho^{2} & \ldots & \rho^{T-1} \\
\rho & 1 & \rho & \ldots & \rho^{T-2} \\
\vdots & & \ddots & & \vdots \\
\rho^{T-2} & \rho^{T-3} & \ldots & 1 & \rho \\
\rho^{T-1} & \rho^{T-2} & \ldots & \rho & 1
\end{array}\right] .
$$

Comme il est nécessaire que $0<\sigma_{\eta}^{2}<1$ (car $\sigma_{\eta}^{2}+\sigma_{\epsilon}^{2}=1$ ), on pourra poser que $\sigma_{\eta}=\left(1+e^{z_{\eta}}\right)^{-1}$, où le paramètre $z_{\eta} \in \mathbb{R}$ remplace $\sigma_{\eta}$ dans les estimations.

On peut remarquer que les paramètres $\sigma_{\nu}$ et $\rho$ sont identifiés à partir des corrélations entre les équations relatives aux dates 1 à $T$ car ces corrélations sont identifiées dans le modèle probit multivarié. D'où, $\sigma_{\epsilon}$ et, finalement, $\sigma_{\eta}$ sont identifiables.

Posons, maintenant, $U_{i}=\left(u_{i 0}, u_{i 1}, \ldots, u_{i T}\right)^{\prime}$. Il vient que $U_{i} \sim N(0, \Omega)$, avec

$$
\Omega=\left[\begin{array}{cccc}
1 & \rho_{0} & \cdots & \rho_{0} \\
\rho_{0} & & & \\
\vdots & & \Omega_{u} & \\
\rho_{0} & & &
\end{array}\right]
$$

où $\rho_{0}$ représente la corrélation entre le terme d'erreur de l'équation initiale et ceux des équations suivantes. En utilisant une argumentation analogue, cette corrélation est identifiable. Il serait possible de considérer une corrélation spécifique entre l'équation initiale et chaque équation ultérieure. Cette spécification n'a pas été retenue compte tenu du nombre important de paramètres qu'elle comporte et par souci d'obtenir une spécification comparable à celle utilisée par Hyslop (1999) pour les États-Unis.

\subsection{Estimation}

Pour chaque date et chaque individu, on observe la décision de participation $y_{i t} \in\{0 ; 1\}$ (où $i=1, \ldots, n$ et $t=0,1, \ldots, T$ ).

La contribution d'un individu $i$ à la fonction de vraisemblance a pour $\operatorname{expression}^{4}$ :

$$
L_{i}(\theta)=\int_{a_{i 0}}^{b_{i 0}} \int_{a_{i 1}}^{b_{i 1}} \ldots \int_{a_{i T}}^{b_{i T}} \phi\left(u_{0}, u_{1}, \ldots, u_{T} ; \Omega\right) d u_{0} d u_{1} \ldots d u_{T},
$$

\footnotetext{
${ }^{3}$ Pour des raisons pratiques, $\sigma_{\eta}$ a été estimé directement dans le cas de données annuelles sans recourir à cette transformation.

${ }^{4}$ Dans l'application qui est réalisée sur le panel européen, l'ordre d'intégration est égal à 8 dans le cas des estimations sur données annuelles et à 96 dans le cas des estimations sur données mensuelles.
} 
où $\phi(. ; \Omega)$ représente la fonction de densité de probabilité de la loi normale centrée de matrice des variances-covariances $\Omega$. Les valeurs des bornes $a_{i t}$ et $b_{i t}$ sont fixées de la façon suivante :

$$
\left\{\begin{array}{l}
a_{i t}=-\infty, \text { si } y_{i t}=0 \text { et } 0 \leq t \leq T \\
b_{i t}=-x_{i t}^{\prime} \beta-\gamma y_{i t-1}, \text { si } y_{i t}=0 \text { et } 1 \leq t \leq T, \\
b_{i 0}=-x_{i 0}^{\prime} \beta_{0}, \text { si } y_{i 0}=0
\end{array}\right.
$$

et

$$
\left\{\begin{array}{l}
a_{i t}=-x_{i t}^{\prime} \beta-\gamma y_{i t-1}, \text { si } y_{i t}=1 \text { et } 1 \leq t \leq T, \\
a_{i 0}=-x_{i 0}^{\prime} \beta_{0}, \text { si } y_{i 0}=1, \\
b_{i t}=+\infty, \text { si } y_{i t}=1 \text { et } 0 \leq t \leq T, .
\end{array}\right.
$$

On peut alors obtenir une estimation du vecteur $\theta$ de paramètres en maximisant la fonction de log vraisemblance simulée :

$$
\hat{\ell}_{N, H}(\theta)=\sum_{i=1}^{N} \ln \left(\frac{1}{H} \sum_{h=1}^{H} \tilde{p}\left(x_{i} ; u_{i}^{h} ; \theta\right)\right),
$$

où les tirages $u_{i}^{h}$ sont spécifiques à l'individu i et sont i.i.d. $(i=1, \ldots, n)$.

Comme la contribution (4) à la fonction de vraisemblance ne peut pas être calculée analytiquement, on simule des tirages $u_{i}^{h}, h=1, \ldots, H$ (voir l'Annexe). Ces tirages sont indépendants et sont réalisés de telle sorte que l'on n'ait pas de rejet. Comme $u_{i}^{h}$ n'est pas tiré, pour des raisons d'efficacité, dans $\mathbb{R}^{T+1}$, il est pondéré par la probabilité $\tilde{p}\left(x_{i} ; u_{i}^{h} ; \theta\right)$ de façon à obtenir une estimation convergente de $L_{i}(\theta)$. Dans l'équation (5), l'expression générique des termes $\tilde{p}\left(x_{i} ; u_{i}^{h} ; \theta\right)$ est définie dans l'équation (7) située dans l'Annexe. On procède de la même façon pour toutes les contributions individuelles à la fonction de vraisemblance.

L'estimateur du maximum de vraisemblance simulé de $\theta$ obtenu en maximisant la fonction (5) est convergent et efficace si $\frac{\sqrt{N}}{H} \longrightarrow 0$ lorsque $N \longrightarrow$ $+\infty$ et $H \longrightarrow+\infty$ (cf., par exemple, Gouriéroux et Monfort (1991), (1993) et (1997)).

\section{Les données}

\subsection{Description de l'échantillon}

Les données que nous utilisons dans cette étude proviennent du Panel européen des ménages de l'INSEE qui constitue la partie française de l'ECHP (European Community Household Panel). Nous utilisons les huit vagues d'enquête de 1994 à 2001. L'échantillon de travail est restreint aux femmes âgées de 20 à 56 ans en 1994 et vivant en couple (mariées ou non). 
Notre panel est cylindré dans le cas où l'observation est réalisée à l'instant de l'enquête sur une base annuelle. Nous avons utilisé aussi, alternativement, les informations mensuelles relatives à l'activité contenue dans ce Panel et, dans ce cas, les données n'ont pas été cylindrées. Pour les observations réalisées sur une base mensuelle, les trajectoires ont été censurées à droite à partir de la première date où on ne dispose pas de la situation de la femme sur le marché du travail. On observe ici la participation au marché du travail au sens du BIT (Bureau International du Travail), c'est-à-dire que la population active féminine comprend les femmes actives occupées (salariées ou non) et celles au chômage. Dans tous les cas, nous avons retenu les trajectoires des femmes dont les valeurs des variables explicatives sont disponibles dans ce Panel.

Nous disposons d'un échantillon de 1543 femmes (cf. tableau 1, colonne $1)^{5}$. L'âge moyen est de 42.50 ans. La répartition est très hétérogène entre les niveaux d'étude. En effet, $51.51 \%$ des femmes ont un niveau d'étude inférieur au baccalauréat, $15.09 \%$ n'ont aucun diplôme, $11.68 \%$ ont le baccalauréat et $21.65 \%$ ont un diplôme supérieur au baccalauréat. Parmi les femmes vivant en couple, $89.46 \%$ sont mariées. Le revenu moyen net du conjoint est de 22800 Euros par an. Le taux de chômage des conjoints est de 8 \%.

L'examen de la distribution du nombre d'années de travail sur les 8 ans de la période laisse entrevoir des différences de propension des femmes à travailler. Cette hétérogénéité peut provenir des caractéristiques observables telles que l'âge, le niveau d'étude, le revenu du conjoint, l'âge et le nombre des enfants. C'est pour cette raison que nous présentons dans les colonnes suivantes du tableau 1 (colonnes 2 à 6 ) les distributions du nombre d'années travaillées selon ces différentes caractéristiques.

La comparaison des deux premières colonnes du tableau 1 ( 8 années de participation vs 0 année de participation) montre une différence d'âge entre les femmes qui participent durant toute la période d'étude (42.09 ans de moyenne d'âge) et celles qui n'ont jamais participé (47.57 ans en moyenne), ce qui correspondrait à un effet de génération.

Conformément aux implications empiriques de la théorie classique de l'offre de travail, plus le niveau d'étude est élevé et plus les femmes participent au marché du travail. En effet, la proportion de femmes non diplômées est plus élevée chez celles qui n'ont jamais participé (30.70\%) que chez celles qui ont été continuellement actives (10.42\%). Le coût d'opportunité de la

\footnotetext{
${ }^{5}$ Pour les observations effectuées sur une base mensuelle, parce que l'échantillon n'est pas cylindré, l'échantillon comprend plus de femmes et le nombre total de trajectoires sur le marché du travail est égal à 4152 .
} 
non-participation étant croissant en fonction du niveau d'étude, les femmes diplômées ont intérêt à participer plus. Cela peut expliquer que seulement $7.72 \%$ des femmes n'ayant jamais été actives soient diplômées du supérieur.

La fécondité de ces deux catégories de femmes semble assez proche avec un taux légèrement plus important (entre 0.1 et $4 \%$ selon les tranches d'âge des enfants) chez les femmes qui ont participé durant toute la période d'étude. La fécondité, en France, relativement importante par rapport au reste de l'Europe, ne semble donc pas être une barrière à la participation des femmes au marché du travail.

En revanche, le mariage semble être associé à une activité moins importante. En effet, la fréquence des femmes mariées chez celles qui n'ont jamais participé est plus importante que celle qui ont toujours participé. Mais il peut s'agir ici d'un effet de structure, les femmes les plus jeunes vivant plus fréquemment que les autres en couple sans être mariées.

Le revenu annuel des conjoints des femmes inactives est en moyenne plus élevé que celui des actives. Ce résultat confirme la théorie classique d'offre de travail selon laquelle la hausse du revenu du conjoint joue négativement sur l'activité économique de la femme. Toujours conformément à la théorie classique de l'offre de travail, on observe que le taux de chômage du conjoint est plus important chez les femmes en couple qui ont participé durant les 8 années de l'étude que chez celles qui n'ont jamais participé durant cette période.

La résidence en région parisienne est plus propice à l'activité des femmes, ce qui est logique compte tenu de la demande de travail plus soutenue dans ce bassin d'emploi. À moins que cela ne soit la propension à participer qui explique la mobilité plus fréquente vers la région parisienne.

Comparons maintenant les colonnes 4 à 6 du tableau 1 qui décrivent les caractéristiques des femmes qui ont changé de statut d'activité durant la période. Les mouvements (entrées/sorties) des femmes sur le marché du travail s'effectuent plus fréquemment entre 36 et 38 ans. Les femmes jeunes sont plus mobiles sur le marché du travail, que ce soit de l'activité vers l'inactivité ou de l'inactivité vers l'activité. Ainsi, les femmes qui ont effectué au moins deux transitions (activité vers inactivité puis inactivité vers activité par exemple) sont en moyenne plus jeunes que les femmes qui ont eu une trajectoire stable durant toute la période étudiée.

Le diplôme favorise l'entrée sur le marché de travail. Par exemple, 25 $\%$ des femmes en couple qui entrent durablement sur le marché de travail ont un diplôme supérieur au baccalauréat contre seulement $7.72 \%$ chez les 
femmes n'ayant jamais participé. Cela est probablement la conséquence des fins d'études. Cependant, le diplôme ne semble pas empêcher les retraits durables du marché du travail. On constate, ainsi, que $26.52 \%$ des femmes qui se retirent durablement du marché de travail ont un diplôme supérieur au baccalauréat.

À un niveau élevé de revenu du conjoint est associé une participation au marché du travail moins fréquente. Les conjoints des femmes qui sortent du marché du travail ont en moyenne un revenu plus élevé que ceux des femmes qui entrent sur le marché du travail. Le taux de chômage des conjoints des femmes qui effectuent plusieurs transitions sur le marché du travail est plus élevé que celui des conjoints des femmes dont la situation sur le marché du travail est stable. Ces observations laissent penser à l'existence d'une éventuelle corrélation positive entre la qualité de l'insertion sur le marché du travail des conjoints et la stabilité des trajectoires des femmes en couple sur ce marché.

Les entrées sur le marché de travail semblent s'effectuer pour une durée longue : $33.33 \%$ des entrants restent actifs sept ans sur les 8 années de la période considérée. Les sorties définitives du marché du travail, par contre, semblent plus importantes en fin de carrière : $34,85 \%$ de ce sorties sont réalisées avec 7 années de participation sur la période d'observation. L'une des raisons de ces mouvements semble être la fécondité comme le montrent les colonnes 5 et 6 du tableau 1. En effet, les femmes qui sortent du marché du travail et celles qui effectuent plusieurs changements de statut ont, en proportion, davantage de jeunes enfants que celles qui participent continûment au marché du travail de 1994 à 2001.

Pour mieux évaluer l'effet de l'âge, de la fécondité et celui du revenu du conjoint, on mesure le taux d'activité des femmes selon ces différentes caractéristiques en décomposant l'âge des personnes et le revenu de leur conjoint en tranche afin de vérifier les résultats obtenus plus hauts (cf. tableau 2). Cela va nous permettre de tester si l'influence de l'âge de la femme et du revenu de son conjoint sur la participation au marché de travail sont linéaires ou pas.

Le tableau 2 donne l'évolution annuelle des taux d'activité des femmes de l'échantillon. Ce taux est de $72.52 \%$ lors de la première période. Il évolue de façon cyclique. Il baisse légèrement durant les trois années suivantes (1995 à 1997), puis augmente légèrement en 1998 (période de conjoncture favorable) avant de décroître à partir de 1999 jusqu'en 2001.

La relation entre l'activité et l'âge varie dans le temps : la participation croît avec l'âge puis décroît ensuite. Le taux d'activité des femmes en couple dont l'âge se situe entre 30 et 45 ans est assez élevé et varie peu au cours de la 
ТАВ. 1 - Caractéristiques de l'échantillon

\begin{tabular}{|c|c|c|c|c|c|c|}
\hline & $\begin{array}{c}\text { Échantillon } \\
\text { total }\end{array}$ & $\begin{array}{c}\text { Toujours } \\
\text { active }\end{array}$ & $\begin{array}{l}\text { Jamais } \\
\text { active }\end{array}$ & $\begin{array}{c}\text { Une } \\
\text { entrée }\end{array}$ & $\begin{array}{c}\text { Une } \\
\text { sortie }\end{array}$ & $\begin{array}{l}\text { Plusieurs } \\
\text { transitions }\end{array}$ \\
\hline Âge moyen & $\begin{array}{l}42.50 \\
(9.63)\end{array}$ & $\begin{array}{l}42.09 \\
(7.91)\end{array}$ & $\begin{array}{l}47.57 \\
(9.66)\end{array}$ & $\begin{array}{c}37.74 \\
(11.16)\end{array}$ & $\begin{array}{l}36.62 \\
(9.58)\end{array}$ & $\begin{array}{c}37.88 \\
(10.27)\end{array}$ \\
\hline Réside à Paris & $\begin{array}{l}13.50 \\
(0.34)\end{array}$ & $\begin{array}{l}15.67 \\
(0.36)\end{array}$ & $\begin{array}{l}10.25 \\
(0.30)\end{array}$ & $\begin{array}{l}15.62 \\
(0.36)\end{array}$ & $\begin{array}{l}13.26 \\
0.34)\end{array}$ & $\begin{array}{l}12.68 \\
(0.33)\end{array}$ \\
\hline Conjoint chômeur & $\begin{array}{c}8,00 \\
(0.27)\end{array}$ & $\begin{array}{c}7.48 \\
(0.26)\end{array}$ & $\begin{array}{c}5.28 \\
(0.22)\end{array}$ & $\begin{array}{l}11.483 \\
(0.32)\end{array}$ & $\begin{array}{c}9.43 \\
(0.29)\end{array}$ & $\begin{array}{l}14.36 \\
(0.35)\end{array}$ \\
\hline Française & $\begin{array}{l}95.97 \\
(0.20)\end{array}$ & $\begin{array}{l}97.12 \\
(0.17)\end{array}$ & $\begin{array}{c}92 \\
(0.27)\end{array}$ & $\begin{array}{l}96.87 \\
(0.17)\end{array}$ & $\begin{array}{l}95.58 \\
(0.21)\end{array}$ & $\begin{array}{l}96.16 \\
(0.19)\end{array}$ \\
\hline Mariée & $\begin{array}{l}89.46 \\
(0.30) \\
\end{array}$ & $\begin{array}{l}89.47 \\
(0.28) \\
\end{array}$ & $\begin{array}{l}94.71 \\
(0.22) \\
\end{array}$ & $\begin{array}{l}75.00 \\
(0.44) \\
\end{array}$ & $\begin{array}{l}77.90 \\
(0.42) \\
\end{array}$ & $\begin{array}{l}81.71 \\
(0.39) \\
\end{array}$ \\
\hline Non diplômée & $\begin{array}{l}15.09 \\
(0.36)\end{array}$ & $\begin{array}{l}10.42 \\
(0.31)\end{array}$ & $\begin{array}{l}30.70 \\
(0.46)\end{array}$ & $\begin{array}{l}13.54 \\
(0.34)\end{array}$ & $\begin{array}{l}14.92 \\
(0.36)\end{array}$ & $\begin{array}{l}12.38 \\
(0.33)\end{array}$ \\
\hline $\begin{array}{l}\text { Diplôme antérieur } \\
\text { au baccalauréat }\end{array}$ & $\begin{array}{l}51.51 \\
(0.50)\end{array}$ & $\begin{array}{l}54.79 \\
(0.50)\end{array}$ & $\begin{array}{l}53.12 \\
(0.50)\end{array}$ & $\begin{array}{l}53.12 \\
(0.50)\end{array}$ & $\begin{array}{l}45.30 \\
(0.50)\end{array}$ & $\begin{array}{l}47.19 \\
(0.50)\end{array}$ \\
\hline Baccalauréat & $\begin{array}{l}11.68 \\
(0.32)\end{array}$ & $\begin{array}{l}12.23 \\
(0.33)\end{array}$ & $\begin{array}{c}8.46 \\
(0.28)\end{array}$ & $\begin{array}{l}8.33 \\
(0.28\end{array}$ & $\begin{array}{l}13.25 \\
(0.34)\end{array}$ & $\begin{array}{l}15.63 \\
(0.36)\end{array}$ \\
\hline $\begin{array}{l}\text { Diplôme postérieur } \\
\text { au baccalauréat }\end{array}$ & $\begin{array}{l}21.65 \\
(0.41) \\
\end{array}$ & $\begin{array}{l}22.56 \\
(0.42) \\
\end{array}$ & $\begin{array}{c}7.72 \\
(0.27) \\
\end{array}$ & $\begin{array}{c}25 \\
(0.44) \\
\end{array}$ & $\begin{array}{l}26.52 \\
(0.44) \\
\end{array}$ & $\begin{array}{l}24.79 \\
(0.43) \\
\end{array}$ \\
\hline $\begin{array}{l}\text { Revenu du conjoint } \\
\text { (en milliers d'Euros 2001) }\end{array}$ & $\begin{array}{c}22.80 \\
(16.44)\end{array}$ & $\begin{array}{c}22.44 \\
(17.49)\end{array}$ & $\begin{array}{c}24.17 \\
(18.16)\end{array}$ & $\begin{array}{c}21.25 \\
(11.30)\end{array}$ & $\begin{array}{c}21.60 \\
(12.55)\end{array}$ & $\begin{array}{c}22.56 \\
(14.60)\end{array}$ \\
\hline $\begin{array}{l}\text { Enfant âgé de } \\
\text { moins de } 3 \text { ans }\end{array}$ & $\begin{array}{l}13.32 \\
(0.34)\end{array}$ & $\begin{array}{c}8.23 \\
(0.27)\end{array}$ & $\begin{array}{c}6.66 \\
(0.25)\end{array}$ & $\begin{array}{l}23.95 \\
(0.43)\end{array}$ & $\begin{array}{l}25.41 \\
(0.44)\end{array}$ & $\begin{array}{l}20.94 \\
(0.40)\end{array}$ \\
\hline $\begin{array}{l}\text { Enfant âgé de } \\
3 \text { ans à } 5 \text { ans }\end{array}$ & $\begin{array}{l}16.71 \\
(0.37)\end{array}$ & $\begin{array}{l}12.29 \\
(0.33)\end{array}$ & $\begin{array}{l}11.85 \\
(0.32)\end{array}$ & $\begin{array}{l}14.58 \\
(0.35)\end{array}$ & $\begin{array}{l}20.99 \\
(0.41)\end{array}$ & $\begin{array}{l}21.24 \\
(0.41)\end{array}$ \\
\hline $\begin{array}{l}\text { Enfant âgé de } \\
6 \text { ans à } 11 \text { ans }\end{array}$ & $\begin{array}{l}30.27 \\
(0.46)\end{array}$ & $\begin{array}{l}30.13 \\
(0.46)\end{array}$ & $\begin{array}{l}26.47 \\
(0.44)\end{array}$ & $\begin{array}{l}28.12 \\
(0.45)\end{array}$ & $\begin{array}{l}28.73 \\
(0.45)\end{array}$ & $\begin{array}{l}23.01 \\
(0.42)\end{array}$ \\
\hline $\begin{array}{l}\text { Enfant âgé de } \\
\text { plus } 12 \text { ans et plus }\end{array}$ & $\begin{array}{l}47.77 \\
(0.50)\end{array}$ & $\begin{array}{l}54.69 \\
(0.49)\end{array}$ & $\begin{array}{l}54.09 \\
(0.50)\end{array}$ & $\begin{array}{l}31.25 \\
(0.46)\end{array}$ & $\begin{array}{l}34.81 \\
(0.48)\end{array}$ & $\begin{array}{l}34.80 \\
(0.48)\end{array}$ \\
\hline $\begin{array}{l}\text { Nombre moyen } \\
\text { d'enfant }\end{array}$ & $\begin{array}{c}1.49 \\
(1.17)\end{array}$ & $\begin{array}{c}1.45 \\
(1.02)\end{array}$ & $\begin{array}{c}1.57 \\
(1.54)\end{array}$ & $\begin{array}{c}1.24 \\
(1.07)\end{array}$ & $\begin{array}{c}1.49 \\
(1.16)\end{array}$ & $\begin{array}{c}1.32 \\
(1.14)\end{array}$ \\
\hline \multicolumn{7}{|l|}{ Années de participation } \\
\hline Aucune & 17.63 & - & 100 & - & - & - \\
\hline Une & 2.98 & - & - & 9.38 & 8.84 & 6.19 \\
\hline Deux & 3.11 & - & - & 5.21 & 8.84 & 7.96 \\
\hline Trois & 3.43 & - & - & 11.46 & 7.18 & 8.55 \\
\hline Quatre & 4.02 & - & - & 12.50 & 9.94 & 9.44 \\
\hline Cinq & 5.51 & - & - & 9.38 & 13.81 & 15.04 \\
\hline Six & 7.19 & - & - & 18.75 & 18.23 & 17.70 \\
\hline Sept & 13.67 & - & - & 33.33 & 33.15 & 35.10 \\
\hline Huit & 42.45 & 100 & - & - & - & - \\
\hline Effectif Total & 1543 & 655 & 272 & 96 & 181 & 339 \\
\hline
\end{tabular}

Source : Panel européen 1994 - 2001, Insee. En pourcentage en colonne, sauf lorsque spécifié. Les écarts-types empiriques sont donnés entre parenthèses. 
ТАВ. 1 - Caractéristiques de l'échantillon (suite)

\begin{tabular}{|c|c|c|c|c|c|c|}
\hline & $\begin{array}{c}\text { Échantillon } \\
\text { total }\end{array}$ & $\begin{array}{c}\text { Toujours } \\
\text { active }\end{array}$ & $\begin{array}{l}\text { Jamais } \\
\text { active }\end{array}$ & $\begin{array}{c}\text { Une } \\
\text { entrée }\end{array}$ & $\begin{array}{c}\text { Une } \\
\text { sortie }\end{array}$ & $\begin{array}{c}\text { Plusieurs } \\
\text { transitions }\end{array}$ \\
\hline \multicolumn{7}{|l|}{$\hat{A} g e$} \\
\hline Moins de 25 ans & $\begin{array}{c}1.55 \\
(0.12)\end{array}$ & $\begin{array}{c}0.78 \\
(0.09)\end{array}$ & $\begin{array}{c}0.60 \\
(0.08)\end{array}$ & $\begin{array}{l}11.46 \\
(0.32)\end{array}$ & $\begin{array}{c}9.39 \\
(0.29)\end{array}$ & $\begin{array}{c}7.37 \\
(0.26)\end{array}$ \\
\hline De 25 à 29 ans & $\begin{array}{c}8.19 \\
(0.27)\end{array}$ & $\begin{array}{c}5.48 \\
(0.23)\end{array}$ & $\begin{array}{c}3.81 \\
(0.19)\end{array}$ & $\begin{array}{l}12.50 \\
(0.33)\end{array}$ & $\begin{array}{l}17.13 \\
(0.38)\end{array}$ & $\begin{array}{l}20.05 \\
(0.40)\end{array}$ \\
\hline De 30 à 34 ans & $\begin{array}{l}13.84 \\
(0.35)\end{array}$ & $\begin{array}{l}12.82 \\
(0.33)\end{array}$ & $\begin{array}{c}7.77 \\
(0.27)\end{array}$ & $\begin{array}{l}21.87 \\
(0.42)\end{array}$ & $\begin{array}{l}21.55 \\
(0.41)\end{array}$ & $\begin{array}{l}16.51 \\
(0.37)\end{array}$ \\
\hline De 35 à 39 ans & $\begin{array}{l}16.92 \\
(0.37)\end{array}$ & $\begin{array}{l}18.59 \\
(0.39)\end{array}$ & $\begin{array}{l}10.98 \\
(0.31)\end{array}$ & $\begin{array}{l}16.66 \\
(0.37)\end{array}$ & $\begin{array}{l}16.02 \\
(0.37)\end{array}$ & $\begin{array}{l}15.63 \\
(0.36)\end{array}$ \\
\hline De 40 à 44 ans & $\begin{array}{l}16.45 \\
(0.37)\end{array}$ & $\begin{array}{l}21.22 \\
(0.41)\end{array}$ & $\begin{array}{l}11.86 \\
(0.32)\end{array}$ & $\begin{array}{l}12.50 \\
(0.33)\end{array}$ & $\begin{array}{l}13.26 \\
(0.34)\end{array}$ & $\begin{array}{c}8.55 \\
(0.28)\end{array}$ \\
\hline De 45 à 49 ans & $\begin{array}{l}16.78 \\
(0.37)\end{array}$ & $\begin{array}{l}22.19 \\
(0.42)\end{array}$ & $\begin{array}{l}16.36 \\
(0.37)\end{array}$ & $\begin{array}{c}2.08 \\
(0.14)\end{array}$ & $\begin{array}{c}9.94 \\
(0.30)\end{array}$ & $\begin{array}{l}15.34 \\
(0.36)\end{array}$ \\
\hline 50 ans et plus & $\begin{array}{l}26.27 \\
(0.44) \\
\end{array}$ & $\begin{array}{l}18.91 \\
(0.39)\end{array}$ & $\begin{array}{l}48.62 \\
(0.50) \\
\end{array}$ & $\begin{array}{l}22.92 \\
(0.42)\end{array}$ & $\begin{array}{l}12.71 \\
(0.33) \\
\end{array}$ & $\begin{array}{l}16.51 \\
(0.37) \\
\end{array}$ \\
\hline \multicolumn{7}{|c|}{$\begin{array}{l}\text { Revenu mensuel } \\
\text { du conjoint } \\
(\text { en Euro 2001) }\end{array}$} \\
\hline Moins de 900 & $\begin{array}{c}8.83 \\
(0.28)\end{array}$ & $\begin{array}{c}6.92 \\
(0.25)\end{array}$ & $\begin{array}{l}11.49 \\
(0.32)\end{array}$ & $\begin{array}{c}8.33 \\
(0.28)\end{array}$ & $\begin{array}{l}11.60 \\
(0.32)\end{array}$ & $\begin{array}{l}10.32 \\
(0.30)\end{array}$ \\
\hline De 900 à 1100 & $\begin{array}{l}10.46 \\
(0.31)\end{array}$ & $\begin{array}{c}9.58 \\
(0.29)\end{array}$ & $\begin{array}{l}10.75 \\
(0.31)\end{array}$ & $\begin{array}{l}12.50 \\
(0.33)\end{array}$ & $\begin{array}{l}10.50 \\
(0.31)\end{array}$ & $\begin{array}{l}11.21 \\
(0.31)\end{array}$ \\
\hline De 1100 à 1500 & $\begin{array}{l}24.66 \\
(0.43)\end{array}$ & $\begin{array}{l}25.97 \\
(0.44)\end{array}$ & $\begin{array}{l}22.79 \\
(0.42)\end{array}$ & $\begin{array}{l}22.91 \\
(0.42)\end{array}$ & $\begin{array}{l}24.31 \\
(0.43)\end{array}$ & $\begin{array}{l}24.78 \\
(0.43)\end{array}$ \\
\hline De 1500 à 2000 & $\begin{array}{l}24.13 \\
(0.43)\end{array}$ & $\begin{array}{l}25.48 \\
(0.44)\end{array}$ & $\begin{array}{l}22.47 \\
(0.42)\end{array}$ & $\begin{array}{l}26.04 \\
(0.44)\end{array}$ & $\begin{array}{l}26.52 \\
(0.44)\end{array}$ & $\begin{array}{l}20.65 \\
(0.41)\end{array}$ \\
\hline De 2000 à 3000 & $\begin{array}{l}20.37 \\
(0.40)\end{array}$ & $\begin{array}{l}22.77 \\
(0.42)\end{array}$ & $\begin{array}{l}16.59 \\
(0.37)\end{array}$ & $\begin{array}{c}21.33 \\
0.41\end{array}$ & $\begin{array}{l}16.02 \\
(0.37)\end{array}$ & $\begin{array}{l}21.23 \\
(0.41)\end{array}$ \\
\hline 3000 et plus & $\begin{array}{l}11.55 \\
(0.32)\end{array}$ & $\begin{array}{c}9.27 \\
(0.29)\end{array}$ & $\begin{array}{l}15.90 \\
(0.36)\end{array}$ & $\begin{array}{c}9.37 \\
(0.29)\end{array}$ & $\begin{array}{l}11.05 \\
(0.31)\end{array}$ & $\begin{array}{l}11.80 \\
(0.32)\end{array}$ \\
\hline
\end{tabular}

Source : Panel européen 1994 - 2001, Insee. En pourcentage en colonne. Les écarts-types empiriques sont donnés entre parenthèses. 
période d'étude. Les femmes plus âgées sont moins fréquemment actives, ce qui peut s'expliquer par les départs en pré-retraite et en retraite. L'influence de l'âge sur l'activité est donc non-linéaire.

On retrouve l'existence d'une forte corrélation positive entre l'activité et le diplôme qui est conforme aux implications empiriques du modèle d'offre de travail. De plus, le taux d'activité des femmes jeunes vivant en couple varie beaucoup plus par rapport à celui des femmes plus âgées, probablement parce qu'elles occupent plus fréquemment des emplois précaires qui sont plus sensibles à la conjoncture économique et qu'elles se retirent plus fréquemment du marché du travail en cas de chômage prolongé.

Le taux d'activité des mères vivant en couple diminue généralement avec le nombre d'enfants. Ce phénomène peut s'expliquer par l'existence de coûts associés à la garde des enfants. Singulièrement, le taux d'activité est plus faible lorsque les enfants sont très jeunes ce qui peut s'expliquer, notamment, par de plus grandes difficultés à trouver des places dans des structures de garde adaptées avant l'âge minimum de scolarisation.

La fréquence d'activité par tranche du revenu du conjoint de la femme mérite d'être analysée de façon plus approfondie. Selon les implications empiriques du modèle classique d'offre de travail, l'augmentation du revenu du ménage autre que celui du travail de la femme - ici le revenu du conjoint - doit être associée à une diminution de l'offre de travail de la femme. Or, nous observons une fréquence d'activité plus faible pour les femmes dont le revenu du conjoint appartient à la première tranche de revenu. L'activité des femmes en couple ne diminue en fonction du revenu du conjoint qu'à partir de la dernière tranche de revenu du conjoint. Nous pouvons donner une explication à ce phénomène. L'effet de la tranche inférieure du revenu du conjoint sur le taux d'activité de la femme peut être expliqué, dans le cadre du système "socio-fiscal", par la perte des droits à certaines allocations lors de la reprise d'activité pour les femmes dont le conjoint a des revenus faibles. En effet, l'éligibilité à certaines allocations est basée, en partie, sur des conditions relatives au revenu total du ménage. Au total, on peut conclure de cette analyse que l'effet du revenu du conjoint sur l'activité de la femme en couple ne peut pas être considéré comme linéaire. Nous opterons donc, dans la modélisation économétrique, pour une spécification non linéaire du revenu du conjoint.

\subsection{Modèle probit simple}

Le tableau 3 donne les résultats d'un modèle probit simple sur l'ensemble de l'échantillon toutes les années confondues, ce qui revient à faire l'hypo- 
TAB. 2 - Taux d'activité des femmes en couple de 1994 à 2001 en \%

\begin{tabular}{|c|c|c|c|c|c|c|c|c|}
\hline & 1994 & 1995 & 1996 & 1997 & 1998 & 1999 & 2000 & 2001 \\
\hline \multicolumn{9}{|l|}{ Âge des enfants } \\
\hline Moins de 3 ans & 68.53 & 60.74 & 58.23 & 52.34 & 56.57 & 58.19 & 60.00 & 60.00 \\
\hline De 3 ans à 5 ans & 66.90 & 61.54 & 61.48 & 60.58 & 62.87 & 62.45 & 68.64 & 68.16 \\
\hline De 6 ans à 11 ans & 70.86 & 66.95 & 68.51 & 65.76 & 68.23 & 69.23 & 70.28 & 71.58 \\
\hline 12 ans et plus & 70.55 & 67.61 & 66.12 & 68.56 & 70.49 & 71.73 & 71.54 & 70.93 \\
\hline \multicolumn{9}{|l|}{ Nombre d'enfants } \\
\hline Aucun Enfant & 74.46 & 67.06 & 66.17 & 61.02 & 62.93 & 59.38 & 53.81 & 48.76 \\
\hline Un & 78.99 & 74.23 & 73.30 & 71.86 & 74.22 & 76.00 & 72.44 & 69.03 \\
\hline Deux & 75.39 & 73.07 & 73.60 & 71.90 & 73.79 & 73.39 & 78.54 & 79.96 \\
\hline Trois et plus & 55.15 & 49.45 & 49.46 & 52.36 & 55.39 & 57.46 & 57.79 & 58.33 \\
\hline \multicolumn{9}{|l|}{ Diplôme } \\
\hline Pas de diplôme & 53.62 & 48.72 & 47.86 & 46.15 & 45.76 & 46.52 & 47.39 & 48.26 \\
\hline Inférieur au baccalauréat & 71.61 & 67.71 & 68.34 & 65.16 & 67.72 & 68.01 & 65.11 & 63.14 \\
\hline Baccalauréat & 78.31 & 75.68 & 70.11 & 71.98 & 74.86 & 71.35 & 72.16 & 72.83 \\
\hline Supérieur au baccalauréat & 85.14 & 78.22 & 78.40 & 78.31 & 80.83 & 79.47 & 81.34 & 76.81 \\
\hline \multicolumn{9}{|l|}{$\begin{array}{l}\text { Revenu mensuel } \\
\text { du conjoint } \\
(\text { en Euro } 2001)\end{array}$} \\
\hline Moins de 900 & 62.31 & 60.33 & 56.76 & 54.55 & 52.78 & 54.90 & 55.00 & 58.25 \\
\hline De 900 à 1100 & 74.52 & 63.40 & 64.33 & 60.56 & 64.86 & 60.77 & 59.26 & 66.17 \\
\hline De 1100 à 1500 & 72.09 & 67.48 & 69.47 & 68.57 & 71.82 & 69.47 & 68.05 & 66.75 \\
\hline De 1500 à 2000 & 75.06 & 70.26 & 67.10 & 66.15 & 69.60 & 71.32 & 68.68 & 72.24 \\
\hline De 2000 à 3000 & 78.46 & 72.62 & 75.79 & 72.62 & 73.17 & 72.35 & 73.31 & 63.98 \\
\hline 3000 et plus & 63.39 & 64.32 & 59.57 & 59.68 & 59.90 & 60.61 & 61.08 & 57.14 \\
\hline Conjoint chômeur & 76.52 & 68.29 & 70.83 & 66.09 & 72.00 & 68.12 & 71.90 & 68.42 \\
\hline \multicolumn{9}{|l|}{$\hat{\text { Age }}$} \\
\hline Moins de 25 ans & 77.63 & 64.41 & 58.82 & 66.67 & 71.43 & 100.00 & - & - \\
\hline De 25 à 29 ans & 76.10 & 70.33 & 70.86 & 59.87 & 64.10 & 62.07 & 57.63 & 58.82 \\
\hline De 30 à 34 ans & 75.40 & 70.24 & 70.82 & 66.02 & 70.00 & 66.83 & 69.23 & 70.29 \\
\hline De 35 à 39 ans & 76.14 & 70.00 & 69.70 & 71.93 & 73.80 & 72.59 & 78.17 & 78.11 \\
\hline De 40 à 44 ans & 79.01 & 74.02 & 76.02 & 76.92 & 75.78 & 81.54 & 80.38 & 77.27 \\
\hline De 45 à 49 ans & 70.88 & 72.14 & 70.85 & 75.66 & 77.91 & 78.97 & 78.35 & 78.86 \\
\hline 50 ans et plus & 56.20 & 53.28 & 53.13 & 49.33 & 53.77 & 53.20 & 49.81 & 47.55 \\
\hline Total en \% & 72,52 & 67,92 & 67,53 & 65,91 & 68,05 & 67,73 & 66,88 & 65,07 \\
\hline
\end{tabular}


thèse, ici, de l'absence d'un effet dynamique.

D'après les résultats des estimations de cette première version du modèle, la participation des femmes est la plus fréquente sur la tranche d'âge 30-44 ans. On peut donner deux explications différentes à cet effet de l'âge selon que l'on s'intéresse aux moins de 30 ans ou aux plus de 50 ans. Cette influence de l'âge sur l'activité des femmes est probablement le résultat, pour les moins de 30 ans, de la conséquence des poursuites d'études, d'une part, et d'une résultante de la fécondité, d'autre part. Nous discuterons un peu plus loin de l'effet de la fécondité. Pour les femmes âgées de 50 ans et plus, l'évolution constatée du taux d'activité est la conséquence d'un effet de générations. Ce résultat est conforme, en particulier, avec ce qui a été déjà mis en évidence précédemment sur les quatre premières vagues du panel européen (cf. Lollivier (2001)) qui montre par des statistiques descriptives, que les femmes nées avant 1950 avaient des taux de participation moins élevés que les générations de femmes ultérieures.

La participation au marché du travail est plus fréquente chez les femmes adultes, de 30 à 44 ans. Aux âges jeunes les femmes poursuivent leurs études ou restent inactives pour des raisons liées à la fécondité. Plus tard, les femmes vivant en couple se retirent de plus en plus fréquemment du marché du travail au fur et à mesure des passages à la retraite.

Plus le niveau de diplôme est élevé, plus les femmes en couple participent au marché du travail. Elles ont un coût d'opportunité associé au loisir plus élevé dans ce cas. Par ailleurs, le mariage semble ne pas avoir d'effet significatif sur la participation. Lorsqu'elles résident en région parisienne, elles participent plus fréquemment au marché du travail, de même lorsqu'elles sont de nationalité française.

Le fait d'avoir des enfants à un impact négatif sur l'activité, ce qui peut s'expliquer par les coûts associés à la présence d'un enfant, coûts d'autant plus élevés que l'enfant est jeune ${ }^{6}$. Ainsi, les coefficients estimés de l'effet du nombre d'enfants sur la participation sont négatifs quel que soit la tranche d'âge. Plus les enfants sont jeunes, plus les valeurs absolues des paramètres estimés associés aux nombre d'enfants sont élevées.

On peut rapprocher ces résultats de ceux obtenus par Hyslop (1999) sur le PSID entre 1979 et 1985, pour les États-Unis. Sur données américaines, l'effet sur l'activité de la présence d'un enfant est plus important lorsque l'enfant est très jeunes (moins de 2 ans) et cet impact est toujours négatif quel

\footnotetext{
${ }^{6}$ Pour un modèle structurel de l'activité des femmes en présence de coûts de participation, on pourra se reporter à Lanot et Robin, 1997.
} 
que soit l'âge de l'enfant. De même, plus récemment, Arellano et Carrasco (2003), à partir de données issues du PSID, trouvent une relation négative entre l'activité et la présence d'enfant dans le ménage, particulièrement pour les enfants les plus jeunes.

En ce qui concerne le revenu du conjoint, les résultats trouvés ne concordent que partiellement avec les implications empiriques de la théorie classique de l'offre de travail. En effet, on s'attend à ce que les femmes dont les conjoints ont de bas revenus participent davantage au marché du travail, ce qui n'est pas complètement vérifié ici. En effet, les femmes dont les conjoints ont un revenu mensuel inférieur à 900 Euros participent au marché du travail moins fréquemment que celles dont le revenu du conjoint est compris entre 1100 et 1500 Euros. En revanche, le fait que le conjoint soit au chômage a un effet significatif et positif sur l'activité de la femme (cf. section précédente).

\section{Résultats du probit dynamique}

On estime deux modèles dynamiques avec effet individuel aléatoire à savoir un probit à effets aléatoires (RE) et un probit à effets aléatoires corrélés (CRE). Dans ces deux modèles, contrairement au modèle probit simple sur données de panel présenté plus haut, nous tenons compte de l'aspect dynamique de la participation en incorporant, dans notre spécification, une relation de dépendance à l'ordre 1. Nous introduisons, dans la modélisation, un effet aléatoire et traitons le problème des conditions initiales (cf. Heckman, 1981). Les deux modèles dynamiques retenus sont estimés sur une base annuelle de 1994 à 2001 (soit 8 ans) et, ensuite, mensuelle. L'examen de ces deux spécifications permet d'affiner l'analyse de la dépendance de l'état.

Les résultats figurent dans le tableaux 4, pour la version annuelle, et dans les tableaux 5 et 6 , pour la version mensuelle. L'impact de l'âge sur l'activité est significatif pour les femmes âgées de 45 ans et plus et celles âgées de moins de 30 ans (pour les deux modèles). Pour ces deux classes d'âge, l'activité des femmes en couple est moins importante. On retrouve ici ce que nous avons déjà observé pour le modèle probit simple. La poursuite des études et les fréquentes transitions de l'activité vers l'inactivité à l'occasion d'une naissance peuvent expliquer la faible participation des femmes jeunes par rapport à celles appartenant à notre tranche d'âge de référence (35 - 39 ans). Enfin, les sorties de l'emploi sont plus fréquentes à partir de 50 ans. Ces sorties sont souvent définitives et effectuée à l'occasion des départs à la retraite ou en pré-retraite.

On retrouve aussi l'impact positif du diplôme sur la probabilité de par- 
TAB. 3 - Modèle Probit

\begin{tabular}{|c|c|c|}
\hline & Coefficient & Écart-type \\
\hline \multicolumn{3}{|l|}{$\overline{\hat{A} g e}$} \\
\hline Moins de 25 ans & $-0.547^{*}$ & 0.110 \\
\hline De 25 à 29 ans & $-0.266^{*}$ & 0.060 \\
\hline De 30 à 34 ans & -0.022 & 0.049 \\
\hline De 35 à 39 ans & Réf & Réf \\
\hline De 40 à 44 ans & -0.056 & 0.047 \\
\hline De 45 à 49 ans & $-0.320^{*}$ & 0.051 \\
\hline 50 ans et plus & $-1.137^{*}$ & 0.048 \\
\hline \multicolumn{3}{|l|}{ Diplôme } \\
\hline Pas de diplôme & $-0.422^{*}$ & 0.036 \\
\hline Inférieur au baccalauréat & Réf & Réf \\
\hline Baccalauréat & $0.259^{*}$ & 0.042 \\
\hline Supérieur au baccalauréat & $0.521^{*}$ & 0.037 \\
\hline \multicolumn{3}{|l|}{ Revenu mensuel du conjoint } \\
\hline Moins de 900 Euros & $-0.247^{*}$ & 0.050 \\
\hline De 900 à 1100 Euros & -0.020 & 0.046 \\
\hline De 1100 Euros à 1500 Euros & Réf & Réf \\
\hline De 1500 Euros à 2000 Euros & -0.062 & 0.036 \\
\hline De 2000 Euros à 3000 Euros & $-0.099^{*}$ & 0.039 \\
\hline 3000 Euros et plus & $-0.556^{*}$ & 0.048 \\
\hline Conjoint chômeur & $0.134^{*}$ & 0.049 \\
\hline \multicolumn{3}{|l|}{ Fécondité } \\
\hline Aucun Enfant & Réf & Réf \\
\hline Nombre d'enfants de moins de 3 ans & $-0.661^{*}$ & 0.039 \\
\hline Nombre d'enfants de 3 ans à 5 ans & $-0.409^{*}$ & 0.034 \\
\hline Nombre d'enfants de 6 ans à 11 ans & $-0.321^{*}$ & 0.023 \\
\hline Nombre d'enfants de 12 ans et plus & $-0.131^{*}$ & 0.015 \\
\hline \multicolumn{3}{|l|}{ Complément de Biographie } \\
\hline Française & $0.305^{*}$ & 0.064 \\
\hline Paris & $0.324^{*}$ & 0.039 \\
\hline Mariée & 0.026 & 0.045 \\
\hline Constante & $0.984^{*}$ & 0.092 \\
\hline
\end{tabular}

$\left(^{*}\right)$ Significatif à $5 \%$

(**) Significatif à $10 \%$ 
ticipation des femmes en couple. Cependant, il n'y a pas de différence significative au niveau de la participation entre les femmes ayant un diplôme inférieur au baccalauréat et celles ayant obtenu un baccalauréat contrairement à ce que l'on a trouvé à partir d'un modèle probit sans effet individuel. Par conséquent, la détention d'un baccalauréat ne garantit pas l'accès à des rémunérations suffisamment élevées pour inciter les bachelières à entrer plus fréquemment sur le marché du travail et, ceci, relativement à des diplômes nécessitant, pour leur obtention, moins d'années d'étude.

La présence d'un enfant est, ici encore, associée à une fréquence de l'activité plus faible, surtout lorsqu'il est très jeune. Une femme avec un enfant reste plus fréquemment hors du marché du travail, surtout si l'enfant a moins de 3 ans. En effet, la valeur absolue du coefficient associé au nombre d'enfants âgés d'au moins 3 ans est plus grande que les valeurs absolues des paramètres associés aux nombres d'enfants de plus de 3 ans. Comme on l'a remarqué plus haut, ceci peut s'expliquer par l'existence de coûts de participation - frais de garde, par exemple - qu'engendre la présence d'un enfant lorsque les parents travaillent. Cependant, si l'on introduit dans la modélisation de l'hétérogénéité non observable et une relation de dépendance à l'ordre 1 , cet effet diminue rapidement avec l'âge de l'enfant et finit par s'annuler lorsque l'enfant est âgé de 12 ans et plus. On observe que les valeurs absolues des coefficients associés aux variables de fécondité sont plus grandes dans le deuxième modèle que dans le premier (i.e. dans le modèle CRE plutôt que dans le RE). L'effet des enfants est donc plus fort lorsque la femme est supposée "contrôler" - anticiper - parfaitement sa fécondité. Contrairement aux résultats d'Hyslop pour les Etats-Unis, l'introduction des naissances passées et futures dans la modélisation augmente l'effet négatif de la fécondité sur l'activité pour la France. En France, les femmes se retirent plus fréquemment du marché du travail de façon temporaire lorsque l'on contrôle, dans la modélisation, par la fécondité. Ceci peut être expliqué par l'environnement législatif rendant la poursuite d'une activité salarié après une naissance possible (existence de diverses aides ou structures d'accueil).

Dans les deux spécifications dynamiques, nous avons décidé, au vu des résultats obtenus avec le modèle probit simple, de ne plus mesurer l'effet global du revenu du conjoint mais de le décomposer en l'effet d'un choc temporaire sur le revenu et en l'impact d'un choc permanent sur ce même revenu. Pour ce faire, nous décomposons le revenu du conjoint en deux composantes : une transitoire et une permanente. La composante permanente, notée $y_{p i}$, est mesurée par la moyenne des revenus sur toute la période d'étude et, la composante transitoire, notée $y_{m i}$, est l'écart obtenu par rapport à cette moyenne. Dans le modèle CRE (colonne 2, tableau 4), nous supposons que l'individu anticipe parfaitement le futur et, donc, que la femme anticipe donc parfaitement les évolutions aussi bien transitoires que permanentes du revenu de son 
conjoint. Chaque fois qu'elle révise ses préférences, elle tient donc compte des revenus présents et futurs de son conjoint, ce qui n'était pas le cas dans le modèle RE (colonne 1, tableau 4). Dans les deux cas, le revenu transitoire n'a pas d'impact significatif sur l'activité. Seul le revenu permanent réduit l'activité féminine. Cela signifie qu'une augmentation - ou une baisse - temporaire du revenu du conjoint ne modifie pas le choix d'activité de la femme. Seule une hausse permanente du revenu du conjoint pousse les femmes en couple à sortir du marché du travail. Aussi, une politique publique visant à modifier l'offre de travail n'aura d'impact qui si elle modifie le revenu permanent des agents et si elle est bien intégrée dans les anticipations de ces derniers. On rejoint ici la critique de Lucas en insistant sur l'importance de la prise en compte des anticipations des agents et de leur mode de formation pour pouvoir apprécier l'impact des politiques économiques.

Le chômage du conjoint ne conduit pas systématiquement les femmes en couple à participer au marché du travail. Si la durée du chômage du conjoint n'est pas suffisamment importante pour influencer le revenu permanent, en particulier de fait de l'existence d'allocations chômage, la femme n'a aucun intérêt modifier sa décision de participation. Cet effet est d'autant plus net que le revenu du conjoint n'est pas très élevé. En effet, l'entrée sur le marché du travail d'une femme dont le conjoint a un bas revenu peut faire perdre au couple certaines subventions et avantages sociaux tout en engendrant des coûts supplémentaires si le couple a des enfants. De plus, si cette femme n'est pas suffisamment diplômée pour avoir de bonnes chances de trouver un emploi avec un salaire relativement important, alors le gain financier espéré associé au passage de l'inactivité à l'activité peut être nul voire négatif. Dans ce cas, la décision de ne pas participer au marché du travail peut s'expliquer sous une hypothèse de rationalité du comportement.

Il est important de remarquer que la prise en compte de l'hétérogénéité non observable permet d'obtenir une estimation convergente des coefficients du modèle. Ainsi, on peut noter que la première estimation - cas du modèle probit simple - surestime les effets de l'âge, du revenu non salarial et celui du diplôme.

Cette modélisation indique une persistance importante de l'activité et de l'emploi des femmes en couple. Ainsi une femme qui est active une année donnée a de fortes chances d'être encore active l'année suivante. La participation des femmes en couple ne serait donc pas due exclusivement aux seules caractéristiques individuelles observables et inobservables mais aussi et surtout à leurs situations antérieures sur le marché du travail (phénomène de dépendance de l'état). Cette dépendance de l'état mis en évidence empiriquement peut être expliquée par l'existence de coûts de recherche pour les chômeurs. 
Les résultats des estimations confirment que la distribution initiale des individus entre les états (active/inactive) est informative de celle de l'hétérogénéité inobservée $\left(\rho_{0} \neq 0\right)$. D'autre part, la valeur estimée de la corrélation entre le terme d'erreur initial et les termes d'erreur pour les dates ultérieures est positive et significative. Cette composante d'hétérogénéité inobservable reflète essentiellement des composantes permanentes telles que des différences dans les préférences individuelles ou dans le revenu permanent non prises en compte par les caractéristiques observables.

La valeur estimée du coefficient associé à la partie auto-régressive des termes d'erreur est négative et significativement différente de zéro (paramètre $\rho \neq 0$ ). Cela montre que les termes d'erreur de modèle rendent compte de chocs transitoires qui se résorbent assez rapidement.

Sur la base d'un test du rapport de vraisemblances, on ne peut pas rejeter la restriction correspondant à la spécification avec effets aléatoires non corrélés (RE). Par conséquent, on ne peut pas rejeter l'hypothèse que la fécondité et le revenu non salarial ne sont pas corrélés globalement avec la décision de participation. En effet, cette hypothèse d'exogénéité peut être testée en comparant les deux premières colonnes du tableau 4 lorsque l'on modélise l'activité vs l'inactivité. Si on s'intéresse à la participation, le modèle à effet aléatoire non corrélé ne peut pas être raisonnablement rejeté. À la différence de ce qui a été trouvé par Hyslop (1999) pour les États-Unis, lorsque l'on tient compte de l'existence d'une corrélation entre ces variables et l'effet individuel dans le cadre du modèle CRE, l'impact de la présence d'enfants sur la décision de participation est plus fort. Aussi, lorsque l'effet individuel rend compte de la fécondité individuelle globale, une part plus importante de la mobilité des femmes vivant en couple est expliquée par la venue des enfants.

La spécification probit dynamique a été utilisée pour modéliser aussi l'emploi (versus le non emploi). Les résultats des estimations figurent au tableau 4 (colonnes 3 et 4) pour la version annuelle. Les résultats son généralement similaires à ceux obtenus pour la participation au marché du travail. Cependant, la présence d'un enfant de moins de trois ans a un impact négatif mais plus important sur la fréquence de la participation que sur celle de l'emploi. Cela peut signifier qu'il est plus difficile pour une femme de retrouver un emploi lorsqu'elle a des enfants jeunes dans un contexte où les structures de garde sont peu nombreuses ou relativement coûteuses. La fertilité ne peut pas être complètement considérée comme exogène dans le cadre de cette spécification. L'effet du diplôme est plus fort dans le cadre de cette spécification et c'est le cas aussi de la localisation en région parisienne. Le coefficient associé à la variable endogène retardée est plus important dans la spécification relative à l'emploi que dans celle relative à la participation. Ce 
TAB. 4 - Probit dynamique

\begin{tabular}{|c|c|c|c|c|}
\hline & \multicolumn{2}{|c|}{ Activité vs Inactivité } & \multicolumn{2}{|c|}{ Emploi vs Non emploi } \\
\hline & $\mathrm{RE}, \mathrm{AR}(1)$ & CRE,AR(1) & $\mathrm{RE}, \mathrm{AR}(1)$ & $\mathrm{CRE}, \mathrm{AR}(1)$ \\
\hline & $+\mathrm{SD}(1)$ & $+\mathrm{SD}(1)$ & $+\mathrm{SD}(1)$ & $+\mathrm{SD}(1)$ \\
\hline \multicolumn{5}{|l|}{$\hat{\text { Âge }}$} \\
\hline \multirow{2}{*}{ Moins de 25} & $-0.3347^{*}$ & $-0.3898^{*}$ & $-0.3280^{*}$ & $-0.4598^{*}$ \\
\hline & $(0.1509)$ & $(0.1614)$ & $(0.1354)$ & $(0.1436)$ \\
\hline \multirow[t]{2}{*}{ De 25 à 29 ans } & $-0.1817^{*}$ & $-0.2186^{*}$ & $-0.1868^{*}$ & $-0.2565^{*}$ \\
\hline & $(0.0733)$ & $(0.0830)$ & $(0.0739)$ & $(0.0788)$ \\
\hline \multirow{2}{*}{ De 30 à 34 ans } & 0.0010 & -0.0069 & -0.0164 & -0.0376 \\
\hline & $(0.0482)$ & $(0.0649)$ & $(0.0561)$ & $(0.0572)$ \\
\hline De 35 à 39 ans & Réf & Réf & Réf & Réf \\
\hline \multirow{2}{*}{ De 40 à 44 ans } & -0.0561 & -0.0469 & 0.0005 & 0.0247 \\
\hline & $(0.0601)$ & $(0.0635)$ & $(0.0536)$ & $(0.0561)$ \\
\hline \multirow{2}{*}{ De 45 à 49 ans } & $-0.2234^{*}$ & $-0.1856^{*}$ & $-0.1247^{*}$ & -0.0712 \\
\hline & $(0.0690)$ & $(0.0738)$ & $(0.0645)$ & $(0.0690)$ \\
\hline \multirow[t]{2}{*}{50 ans et plus } & $-0.7791^{*}$ & $-0.7386^{*}$ & $-0.6185^{*}$ & $-0.5621^{*}$ \\
\hline & $(0.0672)$ & $(0.0730)$ & $(0.0699)$ & $(0.0754)$ \\
\hline \multicolumn{5}{|l|}{ Diplôme } \\
\hline \multirow[t]{2}{*}{ Pas de diplôme } & $-0.3061^{*}$ & $-0.2952^{*}$ & $-0.3837^{*}$ & $-0.3686^{*}$ \\
\hline & $(0.0603)$ & $(0.0612)$ & $(0.0657)$ & $(0.0662)$ \\
\hline Inférieur au baccalauréat & Réf & Réf & Réf & Réf \\
\hline \multirow[t]{2}{*}{ Baccalauréat } & 0.0918 & 0.1080 & $0.1540^{*}$ & $0.1868^{*}$ \\
\hline & $(0.0633)$ & $(0.0629)$ & $(0.0702)$ & $(0.0708)$ \\
\hline \multirow[t]{2}{*}{ Supérieur au baccalauréat } & $0.2928^{*}$ & $0.2911^{*}$ & $0.4182^{*}$ & $0.4060^{*}$ \\
\hline & $(0.0539)$ & $(0.0548)$ & $(0.0589)$ & $(0.0601)$ \\
\hline \multicolumn{5}{|l|}{ Revenu du Conjoint } \\
\hline \multirow[t]{2}{*}{$y_{m i}$} & 0.0457 & 0.0421 & 0.0070 & 0.0068 \\
\hline & $(0.0277)$ & $(0.0290)$ & $(0.0237)$ & $(0.0245)$ \\
\hline \multirow[t]{2}{*}{$y_{p i}$} & $-0.0554^{*}$ & $-0.0580^{*}$ & $-0.0719^{*}$ & $-0.0813^{*}$ \\
\hline & $(0.0138)$ & $(0.0147)$ & $(0.0155)$ & $(0.0168)$ \\
\hline \multirow[t]{2}{*}{ Conjoint chômeur } & 0.1118 & 0.1059 & 0.0147 & 0.0018 \\
\hline & $(0.0623)$ & $(0.0630)$ & $(0.0549)$ & $(0.0582)$ \\
\hline
\end{tabular}

Les écarts-type sont entre parenthèses.

(*) Significatif à $5 \%$

(**) Significatif à $10 \%$ 
TAB. 4 - Probit dynamique (suite)

\begin{tabular}{|c|c|c|c|c|}
\hline & \multicolumn{2}{|c|}{ Activité vs Inactivité } & \multicolumn{2}{|c|}{ Emploi vs Non emploi } \\
\hline & $\mathrm{RE}, \mathrm{AR}(1)$ & $\mathrm{CRE}, \mathrm{AR}(1)$ & $\mathrm{RE}, \mathrm{AR}(1)$ & CRE,AR(1) \\
\hline & $+\mathrm{SD}(1)$ & $+\mathrm{SD}(1)$ & $+\mathrm{SD}(1)$ & $+\mathrm{SD}(1)$ \\
\hline \multicolumn{5}{|l|}{ Fécondité } \\
\hline Aucun Enfant & Réf & Réf & Réf & Réf \\
\hline \multirow[t]{2}{*}{ Nombre d'enfants de moins de 3 ans } & $-0.6456^{*}$ & $-0.7186^{*}$ & $-0.5724^{*}$ & $-0.6263^{*}$ \\
\hline & $(0.0476)$ & $(0.0643)$ & $(0.0446)$ & $(0.0554)$ \\
\hline \multirow[t]{2}{*}{ Nombre d'enfants de 3 ans à 5 ans } & $-0.1463^{*}$ & $-0.1992^{*}$ & $-0.1841^{*}$ & $-0.2391^{*}$ \\
\hline & $(0.0449)$ & $(0.0636)$ & $(0.0401)$ & $(0.0526)$ \\
\hline \multirow[t]{2}{*}{ Nombre d'enfants de 6 ans à 11 ans } & $-0.1480^{*}$ & $-0.1924^{*}$ & $-0.1280^{*}$ & $-0.1807^{*}$ \\
\hline & $(0.0326)$ & $(0.0561)$ & $(0.0311)$ & $(0.0473)$ \\
\hline \multirow[t]{2}{*}{ Nombre d'enfants de 12 ans et plus } & -0.0413 & -0.0503 & -0.0370 & -0.0327 \\
\hline & $(0.0231)$ & $(0.0459)$ & $(0.0235)$ & $(0.0393)$ \\
\hline \multicolumn{5}{|l|}{ Complément Biographie } \\
\hline \multirow[t]{2}{*}{ Française } & $0.2538^{*}$ & $0.2602^{*}$ & $0.3874^{*}$ & $0.3986^{*}$ \\
\hline & $(0.1010)$ & $(0.1004)$ & $(0.1128)$ & $(0.1143)$ \\
\hline \multirow[t]{2}{*}{ Mariée } & 0.0399 & 0.0453 & 0.0762 & 0.0672 \\
\hline & $(0.0622)$ & $(0.0622)$ & $(0.0637)$ & $(0.0648)$ \\
\hline \multirow[t]{2}{*}{ Paris } & $0.1949^{*}$ & $0.1905^{*}$ & $0.2330^{*}$ & $0.2549^{*}$ \\
\hline & $(0.0606)$ & $(0.0601)$ & $(0.0656)$ & $(0.0662)$ \\
\hline \multicolumn{5}{|l|}{ Paramètres du Modèle } \\
\hline \multirow[t]{2}{*}{ Constante } & $-0.4375^{*}$ & $-0.4990 *$ & $-0.2476^{* *}$ & $-0.2708^{* *}$ \\
\hline & $(0.1529)$ & $(0.1629)$ & $(0.1478)$ & $(0.1529)$ \\
\hline \multirow[t]{2}{*}{$y_{t-1}$} & $1.9332^{*}$ & $1.9952^{*}$ & $1.1436^{*}$ & $1.1745^{*}$ \\
\hline & $(0.1282)$ & $(0.1444)$ & $(0.0952)$ & $(0.0958)$ \\
\hline \multirow[t]{2}{*}{$\sigma_{\eta}$} & $0.4601^{*}$ & $0.4224^{*}$ & $0.6665^{*}$ & $0.6565^{*}$ \\
\hline & $(0.0558)$ & $(0.0665)$ & $(0.0304)$ & $(0.0313)$ \\
\hline \multirow[t]{2}{*}{$\rho$} & $-0.3642^{*}$ & $-0.3503^{*}$ & $-0.2427^{*}$ & $-0.2612^{*}$ \\
\hline & $(0.0329)$ & $(0.0337)$ & $(0.0387)$ & $(0.0377)$ \\
\hline \multirow[t]{2}{*}{$\rho_{0}$} & $0.3641^{*}$ & $0.3488^{*}$ & $0.5679^{*}$ & $0.5432^{*}$ \\
\hline & $(0.0512)$ & $(0.0569)$ & $(0.0433)^{*}$ & $(0.0427)$ \\
\hline Logarithme de la vraisemblance & -3983.59 & -3968.89 & -4569.01 & -4544.01 \\
\hline \multicolumn{5}{|l|}{ Test du rapport de vraisemblances } \\
\hline$H_{0}: \operatorname{RE} \quad\left(\xi_{R} \sim \chi^{2}(38)\right)$ & - & 29.4 & - & 50.0 \\
\hline Probabilité de dépasser $\xi_{R}$ sous $H_{0}$ & - & 0.8400 & - & 0.0920 \\
\hline
\end{tabular}

Les écarts-type sont entre parenthèses.

(*) Significatif à $5 \%$

(**) Significatif à $10 \%$ 
résultat témoigne que les trajectoires passant par l'emploi sont plus stables.

Nous avons estimé une version mensuelle du modèle dynamique à effet aléatoire (RE). Les résultats figurent au tableau 5 (active vs inactive) et au tableau 6 (emploi vs non emploi). On retrouve les principaux résultats précédemment énoncés dans le cadre du modèle à effet aléatoire. Le coefficient de la variable endogène retardée est plus grand lorsque l'on passe à une échelle de temps plus fine (le mois).

\section{Conclusion}

Dans cet article nous étudions les déterminants et les caractéristiques de la décision de participation au marché du travail des femmes en couple. Nous montrons l'intérêt de recourir à une spécification dynamique à effet individuel aléatoire pour étudier le choix d'activité. En effet, certaines caractéristiques individuelles dont dépendent les préférences individuelles sont non observables. Aussi, il a été nécessaire de modéliser ces composantes d'hétérogénéité dans le but d'obtenir des estimateurs sans biais des paramètres du modèle. Nos résultats montrent d'ailleurs que le modèle sans effet aléatoire - i.e. le modèle probit simple - surestime les effets de l'âge, celui du revenu du conjoint et l'impact du diplôme sur la décision d'activité des femmes.

Les résultats obtenus en estimant la spécification dynamique permettent de conclure à l'existence d'une persistance importante de la décision d'activité d'une année à l'autre. Nous trouvons aussi que les termes d'erreur sont corrélés négativement. Cette corrélation estimée est faible indiquant que les chocs, notamment ceux sur le revenu non salarial, sont transitoires. Nous montrons enfin que les conditions initiales sont fortement informatives de la distribution de l'hétérogénéité non observable, ce qui justifie leur modélisation spécifique.

Par ailleurs, nous retrouvons les résultats usuels concernant la participation féminine au marché du travail comme, d'une part, l'impact positif du niveau du diplôme sur la participation, d'autre part l'effet négatif sur l'activité de la femme de la présence d'un jeune enfant dans le ménage. La spécification dynamique retenue nous permet de révéler l'importance de la composante permanente de l'hétérogénéité et l'importance, dans la décision d'activité, du revenu permanent.

Dans cet article, nous étudions la participation des femmes au marché du travail dans un cadre dynamique particulièrement adéquat pour modéliser ce type de relation économique. La pertinence des hypothèses faites concernant l'exogénéité commune de la fécondité et du revenu du conjoint a été considérée. Cette hypothèse d'exogénéité n'est pas, en effet, rejetée. 
D'autres pistes de recherche consisteront en l'analyse, dans le cadre de cette modélisation dynamique de l'activité, de l'impact la taxation fiscale (cf. Lanot et Robin (1997), Laroque et Salanié (2003)). On pourrait, aussi, chercher à prendre en compte, dans un cadre dynamique, les choix collectifs d'activité dans le ménage (cf. Chiappori, Fortin et Lacroix (2001)). Une modélisation structurelle de l'activité et du volume horaire de travail est également envisageable (cf. Heckman et Macurdy (1980, 1982), Lanot et Robin (1997)) au prix d'hypothèses additionnelles sur le comportement des individus. Enfin, la décision d'activité pourrait être étudiée, à partir de ces mêmes données du Panel européen, en relation avec la mobilité et les salaires (cf. Beffy, Kamionka, Kramarz et Robert (2004), Beffy, Buchinsky, Fougère, Kamionka, Kramarz (2006)).

\section{Annexe : L'estimation des contributions à la vraisemblance}

La contribution individuelle (4) à la fonction de vraisemblance peut être estimée à l'aide de (voir Gouriéroux et Monfort (1997)) :

$$
\hat{p}_{i}^{H}=\frac{1}{H} \sum_{h=1}^{H} \tilde{p}\left(x_{i} ; u_{i}^{h} ; \theta\right)
$$

où $u_{i}^{h}=\left(u_{i 0}^{h}, u_{i 1}^{h}, \ldots, u_{i T}^{h}\right)^{\prime}$ est un tirage sans rejet du vecteur des termes d'erreur associés aux équations du modèle, $H$ est le nombre de tirages retenu et $\tilde{p}$ est une estimation de la contribution conditionnelle à la fonction de vraisemblance pour un tirage des termes d'erreur.

Soit $U=\Gamma u$ où $\Omega=\Gamma \Gamma^{\prime}$ est la décomposition de Cholesky de la matrice $\Omega, u$ est un tirage réalisé dans la loi $N\left(0_{T+1}, I_{T+1}\right)$ et la matrice $\Gamma$ est triangulaire inférieure. On posera $\Gamma=\left[\Gamma_{j k}\right]$, c'est à dire que $\Gamma_{j k}$ est l'élément générique se trouvant au croisement de la ligne $\mathrm{j}$ et de la colonne $\mathrm{k}$ de la matrice $\Gamma(j, k=1, \ldots,(T+1))$. De plus, posons $\alpha_{i t}=\frac{a_{i t}}{\Gamma_{t t}}, \beta_{i t}=\frac{b_{i t}}{\Gamma_{t t}}$ et $\Gamma_{j k}^{0}=\frac{\Gamma_{j k}}{\Gamma_{j j}}$.

Pour l'individu $i$, le tirage $h$ est formé d'un vecteur colonne de longueur $T+1$ tel que $u_{i}^{h}=\left(u_{i 0}^{h}, u_{i 1}^{h}, \ldots, u_{i T}^{h}\right)^{\prime}$.

Pour déterminer le vecteur $u_{i}^{h}$ à chaque calcul de la fonction de vraisemblance, pour une valeur donnée du vecteur de paramètres, on va procéder itérativement.

Le vecteur $u_{i}^{h}$ est construit de la façon suivante :

- Soit $\tilde{u}_{i 0}^{h} \sim U(0,1)$ et posons

$$
u_{i 0}^{h}=\Phi^{-1}\left[\left(\Phi\left(\beta_{i 0}\right)-\Phi\left(\alpha_{i 0}\right)\right) \tilde{u}_{i 0}^{h}+\Phi\left(\alpha_{i 0}\right)\right]
$$


- Soit $\tilde{u}_{i 1}^{h} \sim U(0,1)$ et posons

$$
\begin{aligned}
u_{i 1}^{h}=\Phi^{-1}\left[\left(\Phi\left(\beta_{i 0}-\Gamma_{21}^{0} u_{i 0}^{h}\right)\right.\right. & \\
& \left.\left.-\Phi\left(\alpha_{i 0}-\Gamma_{21}^{0} u_{i 0}^{h}\right)\right) \tilde{u}_{i 1}^{h}+\Phi\left(\alpha_{i 0}-\Gamma_{21}^{0} u_{i 0}^{h}\right)\right]
\end{aligned}
$$

- Soit $\tilde{u}_{i T}^{h} \sim U(0,1)$ et posons

$$
\begin{aligned}
u_{i T}^{h}=\Phi^{-1}\left[\left(\Phi \left(\beta_{i T}\right.\right.\right. & \left.-\Gamma_{(T+1) T}^{0} u_{i(T-1)}^{h}-\ldots-\Gamma_{(T+1) 1}^{0} u_{i 0}^{h}\right) \\
& \left.-\Phi\left(\alpha_{i T}-\Gamma_{(T+1) T}^{0} u_{i(T-1)}^{h}-\ldots-\Gamma_{(T+1) 1}^{0} u_{i 0}^{h}\right)\right) \tilde{u}_{i T}^{h} \\
& \left.+\Phi\left(\alpha_{i T}-\Gamma_{(T+1) T}^{0} u_{i(T-1)}^{h}-\ldots-\Gamma_{(T+1) 1}^{0} u_{i 0)}^{h}\right)\right]
\end{aligned}
$$

où $\Phi$ est la fonction de répartition de la loi $\mathrm{N}(0,1)$.

On obtient ainsi, séquentiellement, toutes les composantes du vecteur de tirages $u_{i}^{h}$.

Une estimation de la contribution individuelle à la fonction de vraisemblance conditionnelle peut être obtenue à partir de la moyenne empirique des éléments suivants :

$$
\begin{aligned}
& \tilde{p}\left(x_{i} ; u_{i}^{h} ; \theta\right)=\left[\Phi\left(\beta_{i 0}-\Phi\left(\alpha_{i 0}\right)\right]\right. \\
& \times \prod_{k=1}^{T}\left[\Phi\left(\beta_{i k}-\Gamma_{(k+1) k}^{0} u_{i(k-1)}^{h}-\ldots-\Gamma_{(k+1) 1}^{0} u_{i 0}^{h}\right)\right. \\
&\left.\quad-\Phi\left(\alpha_{i k}-\Gamma_{(k+1) k}^{0} u_{i(k-1)}^{h}-\ldots-\Gamma_{(k+1) 1}^{0} u_{i 0}^{h}\right)\right]
\end{aligned}
$$

où $x_{i}$ est le vecteur des variables explicatives pour l'individu $i(i=1, \ldots, n)$ et $h=1, \ldots, H$. 


\section{References}

Arellano, M. et R. Carrasco, 2003, "Binary Choice Panel Data Models With Predetermined Variables", Journal of Econometrics, 115, 125-157.

Beffy, M., Kamionka T., Kramarz F. et Ch. Robert, 2004, "Job Mobility and Wages with Worker and Firm Heterogeneity", mimeo, Crest.

Beffy, M., Buchinsky M., Fougère D., Kamionka T. et Kramarz F., 2006, "The Returns to Seniority in France (and Why they are Lower than in the United States)", Cahiers de Recherche du Crest, 2006-05.

Chiappori, A., Fortin B. et G. Lacroix, 2001, "Marriage Market, Divorce Legislation and Household Labor Supply", Journal of Political Economy, 110 , pp. 37-72.

Eckstein, Z., K.I. Wolpin, 1990, "On the Estimation of Labor Force Participation, Job Search, and Job Matching Models Using Panel Data", in Advances in the Theory and Measurement of Unemployment, Weiss Y. and G. Fishelson eds. New-York, Macmillan.

Fougère, D. et T. Kamionka, 1992, "Un modèle markovien du marché du travail", Annales d'Économie et de Statistique, 27, pp. 149-188.

Fougère, D. et T. Kamionka, 2003, "Bayesian Inference for the Mover Stayer Model in Continuous Time with an Application to the Study of Labor Market transitions", Journal of Applied Econometrics, 18, pp. 697-723.

Geweke, J., 1991, "Efficient Similation from the Multivariate Normal and Student-t Distributions Subject to Linear Constraints ", Computing Science ad Statistics : Proceedings of the Twenty-third Symposium on the Interface. Computing Science and Statistics, American Statistical Association, Alexandria, pp. 571-578.

Gouriéroux, Ch. et A. Monfort, 1991, "Simulated Based in Econometric Models with Heterogeneity", Annales d'Économie et de Statistique, 20-21, 69-107.

Gouriéroux, Ch. et A. Monfort, 1993, "Simulated Based Inference : A Survey with Special Reference to Panel Data Models", Journal of Econometrics, 59, 5-33.

Gouriéroux, Ch. et A. Monfort, 1997, "Simulation-Based Econometric Methods", Core Lecture Series, Oxford : Oxford University Press.

Hajivassilou, V., McFadden D. et P. Ruud, 1992, "Simulation of the Multivariate Normal Orthant Probabilites : Methods and Programs", Cowles Foundation Discussion Paper 1021, Yale University.

Heckman, J. J., 1981, "The Incidental Parameters Problem and the Problem of Initial Conditions in Estimating a Discrete Time-Discrete Data Stochastic Process", in Structural Analysis of Discrete Data, eds. C. Manski et D. McFadden. Cambridge, MA, MIT Press.

Heckman, J. J., 1993, "What has been Learned about Labor Supply in the Past Twenty Years?", American Economic Review, 83, 116-121.

Heckman, J. J. et T. E. MaCurdy, 1980, "A life Cycle Model of Female Labour Supply", Review of Economic Studies, 47, pp. 47-74.

Heckman, J. J. et T. E. MaCurdy, 1982, "Corrigendum on A life Cycle Model of Female Labour Supply", Review of Economic Studies, 49, pp. 659-660. 
Heckman, J. J. et R. J. Willis, 1977, "A Beta Logistic Model for the Analysis of Sequential Labor Force Participation by Married Women", Journal of Political Economy, 85, pp. 27-58.

Heckman, J. J. et R. J. Willis, 1979, "Reply to Mincer and Ofek", Journal of Political Economy, 87, pp. 203-211.

Hyslop, D. R., 1999, "State Dependence, Serial Correlation And Heterogeneity in Intertemporal Labor Force Participation of Married Women", Econometrica, 67, pp. 1255-1294.

Kamionka, T., 1998, "SML Estimation in Transition Models", The Econometrics Journal, 1, pp. C129-C153.

Keane, M., 1994, "A Computationally Practical Simulation Estimator for Panel Data", in Econometrica, vol. 62, pp. 95-116.

Lanot, G. et J.M. Robin, 1997, "Participation des femmes au marché du travail en présence de taxation directe et de coûts de participation", Annales d'Économie et de Statistique, 48, 1-14.

Laroque, G. et B. Salanié, 2003, Institutions et emploi : les femmes et le marché du travail en France, Economica, Paris.

Lollivier, S., 1995, "Activité des femmes mariées et hétérogénéité : estimation sur données de panel", Annales d' Économie et de Statistique, 39, pp. 93106.

Lollivier, S., 2001, "Les choix d'activité des femmes en couple : une approche longitudinale", Économie et Statistique, 349-350, pp. 125-140.

Mincer, J. et H. Ofek, 1979, "The Distribution of Life-Time Labor Force Paticipation of Married Women : Comment", Journal of Political Economy, 87, 197-201. 
TAB. 5 - Probit dynamique (active versus inactive)

\begin{tabular}{|c|c|c|c|c|}
\hline & \multicolumn{2}{|c|}{ Équation initiale } & \multicolumn{2}{|c|}{ Équation dynamique } \\
\hline & coefficient & écart-type & coefficient & écart-type \\
\hline \multicolumn{5}{|l|}{$\hat{\text {Agge }}$} \\
\hline Moins de 25ans & $-0.8424^{*}$ & 0.0816 & $-0.2481^{*}$ & 0.0290 \\
\hline De 25 à 29 ans & $-0.2513^{*}$ & 0.0770 & $-0.0946^{*}$ & 0.0229 \\
\hline De 30 à 34 ans & -0.0777 & 0.0737 & -0.0147 & 0.0190 \\
\hline De 35 à 39 ans & Réf & Réf & Réf & Réf \\
\hline De 40 à 44 ans & $-0.0847^{*}$ & 0.0803 & -0.0111 & 0.0182 \\
\hline De 45 à 49 ans & $-0.3463^{*}$ & 0.0839 & $-0.1350^{*}$ & 0.0222 \\
\hline 50ans et plus & $-0.4140^{*}$ & 0.0836 & $-0.3744^{*}$ & 0.0233 \\
\hline \multicolumn{5}{|l|}{ Diplôme } \\
\hline Pas de diplôme & $-0.3323^{*}$ & 0.0605 & $-0.1800^{*}$ & 0.0219 \\
\hline Inférieur au baccalauréat & Réf & Réf & Réf & Réf \\
\hline Baccalauréat & $0.1244^{*}$ & 0.0632 & 0.0407 & 0.0236 \\
\hline Supérieur au baccalauréat & 0.0062 & 0.0544 & $0.1309^{*}$ & 0.0205 \\
\hline \multicolumn{5}{|l|}{$\begin{array}{l}\text { Revenu mensuel } \\
\text { du conjoint }\end{array}$} \\
\hline Moins de 884 Euros & -0.0737 & 0.0533 & -0.0304 & 0.0179 \\
\hline De 884 Euros à 1082 Euros & -0.0170 & 0.0584 & -0.0190 & 0.0174 \\
\hline De 1082 Euros à 1448 Euros & Réf & Réf & Réf & Réf \\
\hline De 1448 Euros à 2028 Euros & -0.0092 & 0.0535 & -0.0094 & 0.0151 \\
\hline De 2028 Euros à 3018 Euros & 0.0174 & 0.0630 & $-0.0363^{*}$ & 0.0180 \\
\hline 3018 et plus & -0.1062 & 0.0782 & $-0.1235^{*}$ & 0.0232 \\
\hline Conjoint chômeur & -0.0020 & 0.0504 & -0.0118 & 0.0163 \\
\hline \multicolumn{5}{|l|}{ Fécondité } \\
\hline Aucun Enfant & Réf & Réf & Réf & Réf \\
\hline Moins de 3 ans & $-0.1292^{*}$ & 0.0419 & $-0.2720^{*}$ & 0.0112 \\
\hline De 3 ans à 5 ans & $-0.1826^{*}$ & 0.0468 & $-0.1393^{*}$ & 0.0127 \\
\hline De 6 ans à 11 ans & $-0.2156^{*}$ & 0.0335 & $-0.1078^{*}$ & 0.0106 \\
\hline 12 ans et plus & $-0.1195^{*}$ & 0.0270 & $-0.0608^{*}$ & 0.0079 \\
\hline
\end{tabular}

(*) Significatif à $5 \%$ 
TAB. 5 - Probit dynamique (active versus inactive, suite)

\begin{tabular}{|c|c|c|c|c|}
\hline & \multicolumn{2}{|c|}{ Équation initiale } & \multicolumn{2}{|c|}{ Équation dynamique } \\
\hline & coefficient & écart-type & coefficient & écart-type \\
\hline \multicolumn{5}{|l|}{ Mois } \\
\hline Janvier & & & $0.1016^{*}$ & 0.0235 \\
\hline Février & & & $0.1782^{*}$ & 0.0238 \\
\hline Mars & & & $0.1522^{*}$ & 0.0242 \\
\hline Avril & & & $0.1865^{*}$ & 0.0244 \\
\hline Mai & & & $0.1534^{*}$ & 0.0220 \\
\hline Juin & & & $0.1827^{*}$ & 0.0228 \\
\hline Juillet & & & $0.3345^{*}$ & 0.0221 \\
\hline Août & & & $0.2364^{*}$ & 0.0262 \\
\hline Septembre & & & $0.0434^{*}$ & 0.0194 \\
\hline Octobre & & & Réf & Réf \\
\hline Novembre & & & $0.1150^{*}$ & 0.0128 \\
\hline Décembre & & & $0.5342^{*}$ & 0.0185 \\
\hline \multicolumn{5}{|c|}{$\begin{array}{l}\text { Complément de } \\
\text { biographie }\end{array}$} \\
\hline Française & $0.0593^{*}$ & 0.0481 & $0.1925^{*}$ & 0.0338 \\
\hline Paris & $0.2447^{*}$ & 0.0611 & $0.1130^{*}$ & 0.0203 \\
\hline Mariée & 0.6469 & 0.1236 & 0.0017 & 0.0171 \\
\hline Constante & $0.6469 *$ & 0.1236 & $-1.1552^{*}$ & 0.0457 \\
\hline$y_{t-1}$ & & & $2.6749^{*}$ & 0.0167 \\
\hline$\sigma_{\eta}$ & & & $0.1853^{*}$ & 0.0312 \\
\hline$\rho$ & & & $-0.3851^{*}$ & 0.0070 \\
\hline$\rho_{0}$ & & & $0.4190^{*}$ & 0.0097 \\
\hline
\end{tabular}

(*) Significatif à $5 \%$ 
ТАВ. 6 - Probit dynamique (emploi versus non emploi)

\begin{tabular}{|c|c|c|c|c|}
\hline & \multicolumn{2}{|c|}{ Équation initiale } & \multicolumn{2}{|c|}{ Équation dynamique } \\
\hline & coefficient & écart-type & coefficient & écart-type \\
\hline \multicolumn{5}{|l|}{$\hat{A} g e$} \\
\hline Moins de 25 ans & $-0.8168^{*}$ & 0.0790 & $-0.2870^{*}$ & 0.0290 \\
\hline De 25 à 29 ans & $-0.2918^{*}$ & 0.0720 & $-0.1088^{*}$ & 0.0227 \\
\hline De 30 à 34 ans & -0.0705 & 0.0685 & -0.0152 & 0.0183 \\
\hline De 35 à 39 ans & Réf & Réf & Réf & Réf \\
\hline De 40 à 44 ans & -0.1281 & 0.0720 & -0.0083 & 0.0174 \\
\hline De 45 à 49 ans & $-0.3031^{*}$ & 0.0776 & $-0.0912^{*}$ & 0.0222 \\
\hline 55 ans et plus & $-0.3092^{*}$ & 0.0795 & $-0.3323^{*}$ & 0.0244 \\
\hline \multicolumn{5}{|l|}{ Diplôme } \\
\hline Pas de diplôme & $-0.3022^{*}$ & 0.0602 & $-0.2138^{*}$ & 0.0254 \\
\hline Inférieur au baccalauréat & Réf & Réf & Réf & Réf \\
\hline Baccalauréat & $0.1871^{*}$ & 0.0609 & 0.0379 & 0.0247 \\
\hline Supérieur au baccalauréat & $0.1225^{*}$ & 0.0523 & $0.1683^{*}$ & 0.0208 \\
\hline \multicolumn{5}{|l|}{$\begin{array}{l}\text { Revenu mensuel } \\
\text { du conjoint }\end{array}$} \\
\hline Moins de 884 Euros & $-0.1099 *$ & 0.0530 & -0.0049 & 0.0172 \\
\hline De 884 Euros à 1082 Euros & -0.0645 & 0.0568 & -0.0046 & 0.0155 \\
\hline De 1082 Euros à 1448 Euros & Réf & Réf & Réf & Réf \\
\hline De 1448 Euros à 2028 Euros & -0.0198 & 0.0526 & -0.0041 & 0.0148 \\
\hline De 2028 Euros à 3018 Euros & -0.0306 & 0.0603 & -0.0238 & 0.0184 \\
\hline 3018 Euros et plus & -0.1249 & 0.0768 & $-0.1083^{*}$ & 0.0251 \\
\hline Conjoint chômeur & -0.0676 & 0.0498 & $-0.0350^{*}$ & 0.0152 \\
\hline \multicolumn{5}{|l|}{ Fécondité } \\
\hline Aucun Enfant & Réf & Réf & Réf & Réf \\
\hline Moins de 3 ans & $-0.1662^{*}$ & 0.0442 & $-0.2509^{*}$ & 0.0123 \\
\hline De 3 ans à 5 ans & $-0.1795^{*}$ & 0.0456 & $-0.1302^{*}$ & 0.0128 \\
\hline De 6 ans à 11 ans & $-0.1545^{*}$ & 0.0331 & $-0.1039^{*}$ & 0.0107 \\
\hline 12 ans et plus & $-0.0959^{*}$ & 0.0251 & $-0.0504^{*}$ & 0.0085 \\
\hline
\end{tabular}

(*) Significatif à $5 \%$ 
TAB. 6 - Probit dynamique (emploi versus non emploi, suite)

\begin{tabular}{|c|c|c|c|c|}
\hline & \multicolumn{2}{|c|}{ Équation initiale } & \multicolumn{2}{|c|}{ Équation dynamique } \\
\hline & coefficient & écart-type & coefficient & écart-type \\
\hline \multicolumn{5}{|l|}{ Mois } \\
\hline Janvier & & & $0.1079^{*}$ & 0.0198 \\
\hline Février & & & $0.0359^{*}$ & 0.0185 \\
\hline Mars & & & $0.0834^{*}$ & 0.0190 \\
\hline Avril & & & $0.1269^{*}$ & 0.0190 \\
\hline Mai & & & $0.0927^{*}$ & 0.0189 \\
\hline Juin & & & $0.1557^{*}$ & 0.0190 \\
\hline Juillet & & & $0.1416^{*}$ & 0.0191 \\
\hline Août & & & $0.1181 *$ & 0.0190 \\
\hline Septembre & & & $0.1280^{*}$ & 0.0196 \\
\hline Octobre & & & Réf & Réf \\
\hline Novembre & & & 0.0123 & 0.0178 \\
\hline Décembre & & & $0.3759^{*}$ & 0.0182 \\
\hline \multicolumn{5}{|c|}{$\begin{array}{l}\text { Compléments de } \\
\text { biographie }\end{array}$} \\
\hline Française & $0.3053^{*}$ & 0.0908 & $0.2193^{*}$ & 0.0386 \\
\hline Paris & $0.3319^{*}$ & 0.0594 & $0.1426^{*}$ & 0.0239 \\
\hline Mariée & 0.1410 & 0.0472 & $0.0482^{*}$ & 0.0177 \\
\hline Constante & $0.4160^{*}$ & 0.1219 & $-1.1437^{*}$ & 0.04998 \\
\hline$y_{t-1}$ & & & $2.4697^{*}$ & 0.0188 \\
\hline$\sigma_{\eta}$ & & & $0.0811^{*}$ & 0.0311 \\
\hline$\rho$ & & & $-0.3474^{*}$ & 0.0060 \\
\hline$\rho_{0}$ & & & $0.4240 *$ & 0.0109 \\
\hline
\end{tabular}

(*) Significatif à $5 \%$ 